\title{
Massive core parameters from spatially unresolved multi-line observations ${ }^{\star}$
}

\author{
V. Ossenkopf, C. Trojan, and J. Stutzki \\ Physikalisches Institut, Universität zu Köln, Zülpicher Straße 77, 50937 Köln, Germany
}

Received 15 November 2000 / Accepted 18 June 2001

\begin{abstract}
We present observations of 15 massive cores in three different CS transitions from the FCRAO $14 \mathrm{~m}$ and the KOSMA $3 \mathrm{~m}$ telescope. We derive physical parameters of these cores using different approaches to the line radiative transfer problem. The local radiative transfer approximations fail to provide reliable values except for the column densities. A self-consistent explanation of the observed line profiles is only possible when taking density gradients and an internal turbulent structure of the cores into account. The observational data can be fitted by a spherically symmetric radiative transfer model including such gradients and a turbulent clumping. We find that the observed massive cores are approximately virialised with a clumpy density profile that decays with a radial exponent of about -1.6 down to a relatively sharp outer boundary. We show that a careful analysis of spatially unresolved multi-line observations using a physical radiative transfer model can provide values for physical parameters that could be obtained otherwise only by direct observations with much higher spatial resolution. This applies to all quantities directly affecting the line excitation, like the mass and size of dense cores. Information on the exact location or number of clumps, of course, always has to rely on high-resolution observations e.g. from interferometers.
\end{abstract}

Key words. line: profiles - radiative transfer - ISM: clouds - ISM: structure - radio lines: ISM

\section{Introduction}

Whereas the average density in molecular clouds falls between about 50 and $1000 \mathrm{~cm}^{-3}$, observations of highdipole-moment molecules like $\mathrm{CS}$ or $\mathrm{NH}_{3}$ reveal cores with densities up to $10^{6} \mathrm{~cm}^{-3}$. Massive cores are typically somewhat warmer than their embedding molecular cloud and show sizes of about $1-3$ pc and masses between some ten and some thousand solar masses. They appear as relatively bright objects in molecular line maps so that they are favoured objects from the viewpoint of the observations. The observed line widths of $2-15 \mathrm{~km} \mathrm{~s}^{-1}$ are considerably larger than thermal, indicating turbulent motions, possibly induced by outflows from star-forming activity. Many of them are associated with young OB or $\mathrm{T}$ Tauri stars suggesting that massive cores are sites of massive and multiple star formation (cf. Myers 1999). For a better understanding of star formation we have to know the physical parameters within these cores, i.e. the geometrical structure, the density, velocity, and temperature distribution.

\footnotetext{
Send offprint requests to: V. Ossenkopf, e-mail: ossk@ph1.uni-koeln.de

* Appendix A is only available in electronic form at http://www.edpsciences.org
}

Dense cores are best traced by molecules like CS, $\mathrm{HC}_{3} \mathrm{~N}, \mathrm{NH}_{3}$, and $\mathrm{H}_{2} \mathrm{CO}$ characterised by high critical densities even for low transitions. Throughout this paper we will use the term "core" for the dense inner part of a cloud which is directly visible in CS, whereas the rest of the cloud may contribute to the radiative excitation but is mostly visible in CO rather than in CS. We call smaller substructures within the cores "clumps". Metastable transitions of $\mathrm{NH}_{3}$ provide reasonable estimates for the kinetic core temperature and the simultaneous observation of several lines from the same isotope provide combined information both on the density and the temperature structure. Here, we use observations of the $2-1$, $5-4$, and 7-6 transitions of $\mathrm{CS}$ and $\mathrm{C}^{34} \mathrm{~S}$ obtained with the FCRAO $14 \mathrm{~m}$ and the KOSMA $3 \mathrm{~m}$ telescopes to study 15 massive cores and complement our observations with CS data from the literature to derive the physical core parameters.

Plume et al. (1997) observed about 150 massive cores in $\mathrm{CS}$ and $\mathrm{C}^{34} \mathrm{~S}$ using the IRAM $30 \mathrm{~m}$ telescope. With an escape probability approximation they derive relatively similar physical parameters for most cores. We compare their results to the parameters obtained for the cores from our sample and test to which extent these results reflect the constraints provided by the set of lines observed, the 
Table 1. Selected source sample with central positions.

\begin{tabular}{lrr}
\hline \multicolumn{1}{c}{ Source } & \multicolumn{1}{c}{$\alpha_{1950}$} & \multicolumn{1}{c}{$\delta_{1950}$} \\
\hline W49A & $19^{\mathrm{h}} 07^{\mathrm{m}} 50.0^{\mathrm{s}}$ & $09^{\circ} 01^{\prime} 32.0^{\prime \prime}$ \\
W33 & $18^{\mathrm{h}} 11^{\mathrm{m}} 19.8^{\mathrm{s}}$ & $-17^{\circ} 56^{\prime} 21.0^{\prime \prime}$ \\
W51A & $19^{\mathrm{h}} 21^{\mathrm{m}} 25.4^{\mathrm{s}}$ & $14^{\circ} 24^{\prime} 45.0^{\prime \prime}$ \\
W3(OH) & $2^{\mathrm{h}} 23^{\mathrm{m}} 15.4^{\mathrm{s}}$ & $61^{\circ} 38^{\prime} 53.0^{\prime \prime}$ \\
W3 & $2^{\mathrm{h}} 21^{\mathrm{m}} 42.5^{\mathrm{s}}$ & $61^{\circ} 52^{\prime} 22.0^{\prime \prime}$ \\
S255 & $6^{\mathrm{h}} 09^{\mathrm{m}} 58.4^{\mathrm{s}}$ & $18^{\circ} 00^{\prime} 19.0^{\prime \prime}$ \\
S235B & $5^{\mathrm{h}} 37^{\mathrm{m}} 31.8^{\mathrm{s}}$ & $35^{\circ} 40^{\prime} 18.0^{\prime \prime}$ \\
S106 & $20^{\mathrm{h}} 25^{\mathrm{m}} 39.8^{\mathrm{s}}$ & $37^{\circ} 12^{\prime} 46.0^{\prime \prime}$ \\
Serpens & $18^{\mathrm{h}} 27^{\mathrm{m}} 25.0^{\mathrm{s}}$ & $01^{\circ} 12^{\prime} 00.0^{\prime \prime}$ \\
DR21 & $20^{\mathrm{h}} 37^{\mathrm{m}} 14.0^{\mathrm{s}}$ & $42^{\circ} 09^{\prime} 00.0^{\prime \prime}$ \\
Mon R2 & $6^{\mathrm{h}} 05^{\mathrm{m}} 14.0^{\mathrm{s}}$ & $-6^{\circ} 23^{\prime} 00.0^{\prime \prime}$ \\
NGC 2264 & $6^{\mathrm{h}} 38^{\mathrm{m}} 26.0^{\mathrm{s}}$ & $9^{\circ} 32^{\prime} 00.0^{\prime \prime}$ \\
OMC-2 & $5^{\mathrm{h}} 32^{\mathrm{m}} 57.0^{\mathrm{s}}$ & $-5^{\circ} 12^{\prime} 12.0^{\prime \prime}$ \\
$\rho$ Oph A & $16^{\mathrm{h}} 23^{\mathrm{m}} 17.9^{\mathrm{s}}$ & $-24^{\circ} 17^{\prime} 18.0^{\prime \prime}$ \\
NGC 2024 & $5^{\mathrm{h}} 39^{\mathrm{m}} 12.0^{\mathrm{s}}$ & $-1^{\circ} 56^{\prime} 40.0^{\prime \prime}$ \\
\hline
\end{tabular}

limitations of the data analysis or the real physical properties of the cores. To study the influence of the data analysis we compare the escape probability approximation with a self-consistent radiative transfer code computing the excitation conditions in an inhomogenous core with internal turbulence.

In Sect. 2 we provide a short overview on the sample and the observations. Section 3 discusses the traditional way to derive the cloud parameters from the observations. Using the mathematical description of the radiative transfer problem in Appendix A we compute cloud parameters in an escape probability approximation. In Sect. 4 the fully self-consistent radiative transfer code from Appendix B is used to derive the cloud parameters. Section 5 compares the resulting data with parameters obtained from independent observations and discusses implications for the physics of massive cores.

\section{Observations}

\subsection{The sample}

The selection of the sample of massive cores was determined by the need of bright "standard" sources for the SWAS satellite which has a spatial resolution of about 4 arcmin in the frequency range between 490 and $560 \mathrm{GHz}$. For a comparison to data obtained at similar angular resolution we used observations taken with the array receiver of the FCRAO $14 \mathrm{~m}$ telescope at $98 \mathrm{GHz}$ (about 1 arcmin resolution) and performed complementary observations with the $3 \mathrm{~m}$ KOSMA telescope at 245 and $343 \mathrm{GHz}$ where its beam size is approximately 2 arcmin.

The sources were selected from the SWAS source list (Goldsmith et al., priv. comm.) to be observable from the FCRAO and KOSMA and bright enough to be detectable in a reasonable integration time. Table 1 lists the 15 sources selected with their central position.

NGC 2024 is not part of the SWAS sample but we have observed this region as a standard for comparison: it is relatively close $(\approx 450 \mathrm{pc})$ and has been studied already by numerous authors using various techniques. An extended map of the cloud and its environment in CS 2-1 was provided by Lada et al. (1991), and line profiles in four transitions of the main CS isotope are given by Lada et al. (1997). Mezger et al. (1992) have identified seven clumps in NGC 2024 from dust observations using the IRAM $30 \mathrm{~m}$ telescope whereas the FCRAO and KOSMA beams can only distinguish between the two bright clumps FIR3 and FIR5. The data analysis is performed for the position of the brightest clump FIR5. Here, the KOSMA beam also contains weak contributions from FIR4, FIR6, and FIR7.

\subsection{Observational details}

All sources except NGC 2024 were observed in CS 2-1 by Howe (priv. comm.) using the FCRAO $14 \mathrm{~m}$ telescope providing a resolution of $53^{\prime \prime}$. and a main beam efficiency $\eta_{\mathrm{mb}}^{2-1}=0.58$. The cores were covered by 30 -point maps with a sampling of $50^{\prime \prime}$. The CS 2-1 spectra for the southern core in NGC 2024 were taken from Lada et al. (1997).

The CS 5-4 and 7-6 observations used the dual channel KOSMA SIS receiver with noise temperatures of about $95 \mathrm{~K}$ in the $230 \mathrm{GHz}$ branch and $120 \mathrm{~K}$ in the $345 \mathrm{GHz}$ branch. The $3 \mathrm{~m}$ telescope provides a spatial resolution of $110^{\prime \prime}$ in CS 5-4 and 80" in CS 7-6. The default observing mode for all cores were cross scans with a separation of $50^{\prime \prime}$ between subsequent points. Only for NGC 2024 complete $5^{\prime} \times 5^{\prime}$ maps were obtained. At the time of the observations the telescope surface provided main beam efficiencies $\eta_{\mathrm{mb}}^{5-4}=0.54$ and $\eta_{\mathrm{mb}}^{7-6}=0.48$ respectively for the two transitions. For all measurements we use the conservative estimate of about $10 \%$ uncertainty for the main beam efficiency, another $10 \%$ atmospheric calibration uncertainty and add another $5 \%$ for possible drifts etc. As systematic errors they might sum up linearly to a total calibration error of at most $25 \%$.

The FCRAO spectrometer had a channel width of $19.5 \mathrm{kHz}$ corresponding to a velocity spacing of $0.060 \mathrm{~km} \mathrm{~s}^{-1}$. For the broad lines from the sample four velocity channels were binned. The resulting rms falls between 0.2 and $0.45 \mathrm{~K}$. The KOSMA spectra were taken with the medium resolution spectrometer (MRS) and the low resolution spectrometer (LRS) providing channel widths of $167 \mathrm{kHz}$ and $688 \mathrm{kHz}$, respectively. Depending on the different combinations of these backends with the receivers at 245 and $343 \mathrm{GHz}$ we obtain velocity spacings between 0.15 and $0.84 \mathrm{~km} \mathrm{~s}^{-1}$. The particular spacing is not important for the analysis performed here because none of the lines shows strong spectral substructure. All points were integrated up to a noise limit of $0.1 \mathrm{~K}$ per channel.

\subsection{Results}

For most sources the CS 2-1 maps show an approximately elliptical intensity peak with a weak elongation at scales of a few times the resolution. In W49A, Serpens, DR21, 
Table 2. Measured line parameters at the central core positions.

\begin{tabular}{|c|c|c|c|c|c|c|c|c|c|}
\hline \multirow[b]{2}{*}{ Source } & \multicolumn{3}{|c|}{ CS 2-1 } & \multicolumn{3}{|c|}{ CS 5-4 } & \multicolumn{3}{|c|}{ CS 7-6 } \\
\hline & $\begin{array}{r}T_{\mathrm{mb}} \\
{[\mathrm{K}]}\end{array}$ & $\begin{array}{c}\int T_{\mathrm{mb}} \mathrm{d} v \\
{\left[\mathrm{~K} \mathrm{~km} \mathrm{~s}^{-1}\right]}\end{array}$ & $\begin{array}{c}\Delta v \\
{\left[\mathrm{~km} \mathrm{~s}^{-1}\right]}\end{array}$ & $\begin{array}{c}T_{\mathrm{mb}} \\
{[\mathrm{K}]}\end{array}$ & $\begin{array}{c}\int T_{\mathrm{mb}} \mathrm{d} v \\
{\left[\mathrm{~K} \mathrm{~km} \mathrm{~s}^{-1}\right]}\end{array}$ & $\begin{array}{c}\Delta v \\
{\left[\mathrm{~km} \mathrm{~s}^{-1}\right]}\end{array}$ & $\begin{array}{c}T_{\mathrm{mb}} \\
{[\mathrm{K}]}\end{array}$ & $\begin{array}{c}\int T_{\mathrm{mb}} \mathrm{d} v \\
{\left[\mathrm{~K} \mathrm{~km} \mathrm{~s}^{-1}\right]}\end{array}$ & $\begin{array}{c}\Delta v \\
{\left[\mathrm{~km} \mathrm{~s}^{-1}\right]}\end{array}$ \\
\hline $\mathrm{W}_{49 \mathrm{~A}^{(a)}}$ & 3.77 & 35.17 & $8.75 \pm 0.55$ & 1.54 & 12.61 & $7.69 \pm 0.19$ & 1.23 & 12.15 & $9.28 \pm 1.24$ \\
\hline $\mathrm{W}_{49} \mathrm{~A}^{(b)}$ & 4.34 & 32.76 & $7.10 \pm 0.38$ & 2.04 & 18.70 & $8.62 \pm 0.15$ & 1.06 & 9.52 & $8.43 \pm 1.03$ \\
\hline W33 & 9.74 & 67.24 & $6.49 \pm 0.16$ & 3.76 & 27.40 & $6.84 \pm 0.05$ & 2.94 & 17.44 & $5.57 \pm 0.05$ \\
\hline W51A & 10.86 & 110.51 & $9.56 \pm 0.09$ & 4.99 & 67.89 & $12.78 \pm 0.09$ & 3.38 & 40.63 & $11.28 \pm 0.08$ \\
\hline $\mathrm{W} 3(\mathrm{OH})$ & 5.50 & 26.48 & $4.53 \pm 0.20$ & 2.13 & 10.13 & $4.46 \pm 0.08$ & 1.25 & 5.81 & $4.40 \pm 0.31$ \\
\hline W3 & 7.67 & 38.97 & $4.77 \pm 0.05$ & 1.35 & 7.72 & $5.36 \pm 0.28$ & 1.56 & 8.44 & $5.08 \pm 0.09$ \\
\hline S255 & 8.02 & 21.55 & $2.53 \pm 0.04$ & 2.91 & 10.13 & $3.28 \pm 0.03$ & 1.67 & 5.29 & $2.99 \pm 0.15$ \\
\hline S235B & 6.38 & 16.21 & $2.39 \pm 0.05$ & 0.85 & 2.67 & $2.97 \pm 0.13$ & 0.42 & 1.10 & $2.53 \pm 0.19$ \\
\hline S106 & 3.29 & 8.19 & $2.34 \pm 0.10$ & 0.83 & 2.11 & $2.40 \pm 0.22$ & 0.65 & 2.23 & $3.23 \pm 0.78$ \\
\hline Serpens & 3.74 & 9.36 & $2.35 \pm 0.13$ & 0.67 & 2.52 & $3.59 \pm 0.35$ & 0.39 & 1.68 & $4.11 \pm 0.65$ \\
\hline DR21 & 6.48 & 25.00 & $3.62 \pm 0.10$ & 2.41 & 9.74 & $3.80 \pm 0.18$ & 2.92 & 10.92 & $3.51 \pm 0.06$ \\
\hline Mon R2 & 5.53 & 12.26 & $1.90 \pm 0.16$ & 2.15 & 6.52 & $2.38 \pm 0.27$ & 1.70 & 3.83 & $1.95 \pm 0.19$ \\
\hline NGC 2264 & 5.64 & 21.90 & $3.66 \pm 0.08$ & 2.30 & 9.48 & $3.89 \pm 0.08$ & 1.25 & 5.42 & $3.91 \pm 0.12$ \\
\hline OMC-2 & 5.62 & 9.26 & $1.55 \pm 0.05$ & 1.96 & 5.93 & $2.85 \pm 0.10$ & 0.92 & 1.54 & $1.58 \pm 0.25$ \\
\hline$\rho \mathrm{Oph} \mathrm{A}$ & 4.22 & 11.10 & $2.47 \pm 0.25$ & 2.07 & 2.30 & $1.04 \pm 0.08$ & $<0.2$ & - & - \\
\hline
\end{tabular}

Mon R2, OMC-2, $\rho$ Oph A, and NGC 2024 we can distinguish a second intensity maximum apart from the central position. Table 2 summarises the parameters of the line profiles at the central position for all cores. The majority of line profiles are approximately Gaussian as indicated by integrated line intensities close to the Gaussian value of $1.06 T_{\mathrm{mb}} \Delta v$ in Table 2 . Broad wings are only visible in S255, W33, and DR21.

W49 shows a double-peak structure which has been interpreted e.g. by Dickel \& Auer (1994) as the footprint of large scale collapse. They fitted $\mathrm{HCO}^{+}$line profiles by a spherical collapse model but concluded that additional components are needed to explain the observations. Using the radiative transfer code from Appendix B we have tested their infall model and found that, while reproducing the $\mathrm{HCO}^{+}$profiles, it completely fails to explain the CS observations. The enhanced blue emission characteristic for collapse is visible only in CS 7-6. In CS 2-1 and 5-4 we rather find an enhanced red emission. No spherically symmetric collapse model can explain these observations. Instead of constructing a more complex model we have simply decomposed the emission into two separate components with a relative velocity of $8.5 \mathrm{~km} \mathrm{~s}^{-1}$ in the line of sight denoted as $\mathrm{W}_{49 \mathrm{~A}^{(\mathrm{a})}}$ and $\mathrm{W} 49 \mathrm{~A}^{(\mathrm{b})}$. From the modelling in Sect. 4 it turns out that we cannot even distinguish whether the two components are moving towards each other or apart as long as they do not line up exactly along the line of sight. Hence, we will treat them separately in the following, ignoring any possible interaction. Further observations including other tracers should be included to better resolve the situation.

In NGC 2024 we also mapped the less abundant isotope $\mathrm{C}^{34} \mathrm{~S}$ with KOSMA in addition to the main CS
Table 3. Measured line parameters within NGC 2024.

\begin{tabular}{lclc}
\hline Transition & $\begin{array}{c}T_{\mathrm{mb}} \\
{[\mathrm{K}]}\end{array}$ & $\begin{array}{c}\int T_{\mathrm{mb}} \mathrm{d} v \\
{\left[\mathrm{~K} \mathrm{~km} \mathrm{~s}^{-1}\right]}\end{array}$ & $\begin{array}{c}\Delta v \\
{\left[\mathrm{~km} \mathrm{~s}^{-1}\right]}\end{array}$ \\
\hline $\mathrm{C}^{32} \mathrm{~S} 5-4$ & 5.19 & 11.65 & $2.10 \pm 0.02$ \\
$\mathrm{C}^{32} \mathrm{~S} 7-6$ & 3.63 & 9.13 & $2.38 \pm 0.08$ \\
$\mathrm{C}^{34} \mathrm{~S} 5-4$ & 1.19 & 2.22 & $1.75 \pm 0.09$ \\
$\mathrm{C}^{34} \mathrm{~S} 7-6$ & 0.65 & 0.98 & $1.45 \pm 0.13$ \\
\hline
\end{tabular}

isotope observed in all cores. The observed line parameters are given in Table 3. Lada et al. (1997) provided detailed CS spectra for the southern core at the position of the FIR5. They obtained in the CS 2-1 transition $T_{\mathrm{mb}}=15.4 \mathrm{~K}, \Delta v=1.80 \mathrm{~km} \mathrm{~s}^{-1}$ at $24^{\prime \prime}$ resolution, in CS $5-4 T_{\mathrm{mb}}=9.6 \mathrm{~K}, \Delta v=2.1 \mathrm{~km} \mathrm{~s}^{-1}$ at $30^{\prime \prime}$ resolution, in CS $7-6 T_{\mathrm{mb}}=10.1 \mathrm{~K}, \Delta v=2.7 \mathrm{~km} \mathrm{~s}^{-1}$ at $20^{\prime \prime}$ resolution, and in CS $10-9 T_{\mathrm{mb}}=10.6 \mathrm{~K}, \Delta v=2.1 \mathrm{~km} \mathrm{~s}^{-1}$ at $14^{\prime \prime}$ resolution.

\section{Cloud parameters from the escape probability model}

To interpret the small amount of information contained in observations of at most five transitions showing mainly Gaussian profiles and essentially unresolved, approximately circular symmetric intensity distributions, we need a simple cloud model that is both physically reasonable and characterised by few parameters. An obvious choice is a spherically symmetric model. This geometry reflects early phases and the large scale behaviour of several collapse simulations (e.g. Galli et al. 1999), whereas the 
inner parts of collapsing clouds are probably flattened structures (e.g. Li \& Shu 1997).

Even in spherical geometry there is no simple way to solve the radiative transfer problem relating the cloud parameters to the emitted line intensities (see Appendix A.1). Thus we cannot compute the cloud properties directly from the observations.

\subsection{Application of the escape probability approximation}

A common approach is the escape probability approximation discussed in detail in Appendix A.2. Assuming that all cloud parameters, including the excitation temperatures, are constant within a spherical cloud volume one can derive a simple formalism relating the three parameters kinetic temperature $T_{\text {kin }}$, gas density $n_{\mathrm{H}_{2}}$, and column density of radiating molecules on the scale of the global velocity variation $N_{\mathrm{mol}} / \Delta v$ to the line intensity at the cloud model surface. No assumption on molecular abundances is required.

Since a telescope does not provide a simple pencil beam we have to correct the model surface brightness temperature by the beam filling factor $\eta_{\mathrm{f}}$, given as the convolution integral of the normalised intensity distribution with the telescope beam pattern, to compute the observable beam temperature. Unfortunately, the brightness profile of the source is a non-analytic function where we can only give simple expressions for the central value observed in a beam much smaller than the source or for the integral value observed in a beam much larger than the source (Eqs. (A.9) and (A.11)). For intermediate situations we approximate the beam temperature by starting from both limits and using a beam filling factor given by the convolution integral of two Gaussians. The difference between the two values provides an estimate of the error made in the beam convolution.

To compute the integral we fitted the observed brightness distributions by Gaussians. Most cores are well approximated by slightly elongated Gaussians. W49A, S235B, Serpens, and $\rho$ Oph A show asymmetric scans so that the size determination is somewhat uncertain. The fit error is about $0.3^{\prime}$ for these three sources. For the rest of the cores we obtain typical values of less than $0.2^{\prime}$. The true object size finally follows from the deconvolution of the measured intensity distribution with the telescope beam. The resulting source sizes in $\alpha$ and $\delta$ are given in Table 4. As the geometric mean is sufficient to compute the beam filling factor we do not expect any serious error from the fact that the cross-scans in $\alpha$ and $\delta$ do not necessarily trace the major axes of the brightness distribution. For sources which are considerably smaller than the beam widths of $53^{\prime \prime}, 107^{\prime \prime}$, and $80^{\prime \prime}$, respectively, only a rough size estimate is possible according to the nonlinearity of the deconvolution. This holds for W49A, W3, S235B, and partially S255. Most clouds, however, show an extent of the emission which is close to the beam size.
Table 4. Source size corrected for beam convolution.

\begin{tabular}{lllllll}
\hline \multicolumn{1}{c}{ Source } & \multicolumn{3}{c}{$F W H M$ in $\alpha\left[^{\prime}\right]$} & \multicolumn{3}{c}{$F W H$ in $\delta\left[^{\prime}\right]$} \\
\hline W49A & $2-1$ & $5-4$ & $7-6$ & $2-1$ & $5-4$ & $7-6$ \\
W33 & 1.0 & 1.1 & 0.3 & 1.5 & 1.7 & 1.3 \\
W51A & 1.7 & 1.7 & 1.4 & 1.7 & 1.3 & 1.0 \\
W3(OH) & 2.1 & 1.7 & 1.7 & 2.1 & 2.2 & 1.1 \\
W3 & 1.0 & 0.6 & 0.8 & 1.0 & 0.6 & 0.3 \\
S255 & 1.9 & 1.6 & 1.1 & 1.2 & 0.6 & 1.1 \\
S235B & 1.0 & 2.2 & 1.1 & 0.9 & 0.2 & 0.3 \\
S106 & 3.0 & 1.6 & & 2.5 & 2.2 & \\
Serpens & 2.0 & 2.2 & & 2.1 & 2.3 & \\
DR21 & 3.0 & 2.6 & 1.8 & 1.5 & 1.7 & 1.8 \\
Mon R2 & 3.2 & 3.0 & 1.1 & 3.6 & 3.2 & 1.6 \\
NGC 2264 & 3.2 & 1.7 & 0.6 & 2.9 & 2.1 & \\
OMC-2 & 2.4 & 1.4 & 0.6 & 2.5 & 1.4 & 0.6 \\
$\rho$ Oph A & 1.3 & 3.0 & 2.6 & 1.9 & 2.9 & 2.5 \\
NGC 2024 & 1.1 & 1.1 & 1.3 & 1.3 & 2.5 & 2.3 \\
\hline
\end{tabular}

In general different values are obtained for the spatial FWHMs in the different lines. In Table 4 we find two classes of sources with respect to the variation of the source size depending on the transition observed. Most cores show a monotonic decrease of the visible size when going to higher transitions. This is expected from the picture that higher transitions are only excited in denser and smaller regions. Serpens, $\rho$ Oph A, and NGC 2024, however show the smallest width of the fit in the CS 2-1 transition. This is explained by eye inspecting the $2-1$ maps and corresponding high-resolution observations from the literature where we see that the three sources break up into several clumps which are only separated in the $53^{\prime \prime}$ beam but unresolved in the KOSMA beams. In these cases, we have restricted the analysis to the major core seen in the CS 2-1 maps using its size to compute the beam filling, although this approach introduces a small error in the data analysis by assigning the whole flux measured in the higher transitions to this central core.

Applying the two limits for the beam size treatment (Eqs. (A.9) and (A.11)) using the sizes from Table 4 we find that the resulting gas and column densities are the same within 20\% except for W49A, W3, S235B, and NGC 2264. The first three are small compared to the beams so that the results from Eq. (A.9) have to be rejected and only Eq. (A.11) can be used. For NGC 2264 we cannot provide a simple explanation for the difference so we give a relatively large error bar covering the results from both approximations.

The size of the resulting parameter range in $T_{\mathrm{kin}}, n_{\mathrm{H}_{2}}$, and $N_{\mathrm{CS}} / \Delta v$ is determined by the accuracy of the observations. For two cores it was only possible to set a lower limit to the gas density. Moreover, we were not able to provide any good constraint to the cloud temperature for all sources. Values between about $30 \mathrm{~K}$ and $150 \mathrm{~K}$ are 
possible. Hence, an independent determination of the cloud temperatures is required. Several different methods based on optically thick $\mathrm{CO}, \mathrm{NH}_{3}$ or dust observations are discussed in the literature and we used the values from the references given in Table 5. In addition to these values we also used $50 \mathrm{~K}$ as assumed by Plume et al. (1997) as "standard" temperature in the parameter determination for massive cores.

\subsection{Resulting core parameters}

Table 5 lists the parameters from the escape probability model for all cores. Whereas the column density is well constrained for most clouds, there is a considerable uncertainty in the gas density resulting from the unknown cloud temperature. At the temperature of $50 \mathrm{~K}$ we obtain average values and logarithmic standard deviation factors of

$$
\begin{aligned}
\left\langle n_{\mathrm{H}_{2}}\right\rangle & =7.9 \times 10^{5} & & \times / \div 1.5 \\
\left\langle N_{\mathrm{CS}} / \Delta v\right\rangle & =1.2 \times 10^{14} & & \times / \div 2.8 .
\end{aligned}
$$

From the 71 cores analysed by Plume et al. (1997) assuming this temperature they obtained

$$
\begin{aligned}
\left\langle n_{\mathrm{H}_{2}}\right\rangle & =8.5 \times 10^{5} & & \times / \div 1.7 \\
\left\langle N_{\mathrm{CS}} / \Delta v\right\rangle & =2.5 \times 10^{14} & & \times / \div 3.1 .
\end{aligned}
$$

This good agreement indicates first that both investigations study the same type of clouds visible in the CS transitions. Second, this shows that the static escape probability (Eq. (A.11)) used here and the LVG escape probability (Eq. (A.9)) applied by Plume et al. (1997) differ only marginally as discussed already by Stutzki \& Winnewisser (1985). Third, the observational data from both telescopes give almost equivalent results, i.e. the reliability of the parameters hardly profits from using the 10 times smaller beam of the IRAM telescope when the escape probability approximation is used.

For NGC 2024 we are able to test the consistency of the results from the $\mathrm{CS}$ and the $\mathrm{C}^{34} \mathrm{~S}$ observations. The resulting hydrogen densities agree for both isotopes within $20 \%$ at all temperatures assumed. We obtain $9 \times 10^{6} \mathrm{~cm}^{-3}$ at $25 \mathrm{~K}$ (Mezger et al. 1992) and $3.2 \times 10^{6} \mathrm{~cm}^{-3}$ at $40 \mathrm{~K}$ (Ho et al. 1993). Unfortunately, this is a core where we can only give a lower limit to the column densities. The limits deviate by a factor 13 , which is significantly different from the terrestrial isotopic ratio of 23 but close to the value 10 derived by Mundy et al. (1986) for the isotopic ratio in NGC 2024.

The escape probability model provides a first estimate to the physical parameters but its limitations are obvious. It is definitely not justified to assume constant parameters within the whole cloud. Moreover, several observations are in contradiction to the parameters from the escape probability models. Lada et al. (1997) detected the CS 10-9 transition in NGC 2024 and Plume et al. (1997) observed the 10-9 and 14-13 transitions in S255 and $\mathrm{W} 3(\mathrm{OH})$.

\begin{tabular}{|c|c|c|c|c|c|}
\hline Source & $\begin{array}{c}T_{\text {kin }} \\
{[\mathrm{K}]}\end{array}$ & $\begin{array}{c}n_{\mathrm{H}_{2}} \\
{\left[\mathrm{~cm}^{-3}\right]}\end{array}$ & $\times / \div$ & $\begin{array}{c}N_{\mathrm{CS}} / \Delta v \\
{\left[\mathrm{~cm}^{-2} / \mathrm{km} \mathrm{s}^{-1}\right]}\end{array}$ & $\times / \div$ \\
\hline \multirow[t]{2}{*}{$\mathrm{W}_{49 \mathrm{~A}^{(a)}}$} & $20^{\mathrm{a}}$ & $>7.3 \times 10^{6}$ & & $2.2 \times 10^{14}$ & 1.3 \\
\hline & $50^{\mathrm{a}}$ & $1.3 \times 10^{6}$ & 1.5 & $2.1 \times 10^{14}$ & 1.2 \\
\hline \multirow[t]{2}{*}{$\mathrm{W} 49 \mathrm{~A}^{(b)}$} & $20^{\mathrm{a}}$ & $4.5 \times 10^{6}$ & 1.6 & $2.8 \times 10^{14}$ & 1.3 \\
\hline & $50^{\mathrm{a}}$ & $7.6 \times 10^{5}$ & 1.5 & $2.7 \times 10^{14}$ & 1.2 \\
\hline \multirow[t]{2}{*}{ W33 } & $40^{\mathrm{b}}$ & $1.7 \times 10^{6}$ & 1.7 & $2.3 \times 10^{14}$ & 1.2 \\
\hline & $50^{\mathrm{c}}$ & $1.2 \times 10^{6}$ & 1.6 & $2.3 \times 10^{14}$ & 1.2 \\
\hline \multirow[t]{3}{*}{ W51A } & $20^{\mathrm{a}}$ & $2.1 \times 10^{7}$ & 1.6 & $4.8 \times 10^{14}$ & 1.7 \\
\hline & 50 & $1.1 \times 10^{6}$ & 1.5 & $4.1 \times 10^{14}$ & 1.4 \\
\hline & $57^{\mathrm{a}}$ & $8.9 \times 10^{5}$ & 1.4 & $3.7 \times 10^{14}$ & 1.4 \\
\hline \multirow[t]{2}{*}{$\mathrm{W} 3(\mathrm{OH})$} & $30^{\mathrm{d}}$ & $1.9 \times 10^{6}$ & 1.5 & $1.3 \times 10^{14}$ & 1.2 \\
\hline & 50 & $8.5 \times 10^{5}$ & 1.4 & $1.3 \times 10^{14}$ & 1.2 \\
\hline \multirow[t]{3}{*}{ W3 } & $30^{\mathrm{e}}$ & $7.1 \times 10^{6}$ & 2.9 & $7.4 \times 10^{14}$ & 1.5 \\
\hline & 50 & $9.8 \times 10^{5}$ & 1.8 & $8.1 \times 10^{14}$ & 1.4 \\
\hline & $55^{\mathrm{f}}$ & $7.4 \times 10^{5}$ & 1.6 & $8.3 \times 10^{14}$ & 1.3 \\
\hline \multirow[t]{2}{*}{ S255 } & $40^{\mathrm{g}}$ & $1.3 \times 10^{6}$ & 1.6 & $1.3 \times 10^{14}$ & 1.3 \\
\hline & 50 & $9.3 \times 10^{5}$ & 1.5 & $1.2 \times 10^{14}$ & 1.3 \\
\hline \multirow[t]{2}{*}{$\mathrm{S} 235 \mathrm{~B}$} & $40^{\mathrm{h}}$ & $1.2 \times 10^{6}$ & 1.5 & $3.7 \times 10^{13}$ & 1.3 \\
\hline & 50 & $8.9 \times 10^{5}$ & 1.5 & $3.6 \times 10^{13}$ & 1.3 \\
\hline \multirow[t]{3}{*}{ S106 } & $10^{\mathrm{i}}$ & $>6.3 \times 10^{6}$ & & $4.7 \times 10^{13}$ & 1.4 \\
\hline & $25^{\mathrm{i}}$ & $7.1 \times 10^{5}$ & 1.5 & $3.8 \times 10^{13}$ & 1.4 \\
\hline & 50 & $2.5 \times 10^{5}$ & 1.6 & $3.9 \times 10^{13}$ & 1.4 \\
\hline \multirow[t]{2}{*}{ Serpens } & $25^{\mathrm{j}}$ & $4.6 \times 10^{5}$ & 1.2 & $2.8 \times 10^{13}$ & 1.5 \\
\hline & 50 & $1.9 \times 10^{5}$ & 1.6 & $2.8 \times 10^{13}$ & 1.5 \\
\hline \multirow[t]{2}{*}{ DR21 } & $35^{\mathrm{k}}$ & $1.5 \times 10^{6}$ & 1.7 & $9.3 \times 10^{13}$ & 1.9 \\
\hline & 50 & $9.1 \times 10^{5}$ & 1.6 & $8.7 \times 10^{13}$ & 1.4 \\
\hline \multirow[t]{2}{*}{ Mon R2 } & $25^{1}$ & $1.7 \times 10^{6}$ & 1.8 & $4.5 \times 10^{13}$ & 1.5 \\
\hline & $50^{\mathrm{m}}$ & $6.2 \times 10^{5}$ & 1.7 & $3.7 \times 10^{13}$ & 1.7 \\
\hline \multirow[t]{2}{*}{ NGC 2264} & $25^{\mathrm{n}}$ & $1.4 \times 10^{6}$ & 2.4 & $7.9 \times 10^{13}$ & 2.6 \\
\hline & 50 & $4.7 \times 10^{5}$ & 2.3 & $7.8 \times 10^{13}$ & 2.5 \\
\hline \multirow[t]{3}{*}{ OMC-2 } & $19^{\mathrm{q}}$ & $1.9 \times 10^{6}$ & 1.6 & $6.3 \times 10^{13}$ & 1.4 \\
\hline & $24^{\mathrm{o}, \mathrm{p}}$ & $1.1 \times 10^{6}$ & 1.5 & $6.2 \times 10^{13}$ & 1.4 \\
\hline & 50 & $3.4 \times 10^{5}$ & 1.7 & $6.2 \times 10^{13}$ & 1.4 \\
\hline \multirow[t]{2}{*}{$\rho \mathrm{Oph} \mathrm{A}$} & $25^{\mathrm{r}}$ & $9.5 \times 10^{5}$ & 1.6 & $2.0 \times 10^{13}$ & 1.4 \\
\hline & 50 & $3.7 \times 10^{5}$ & 1.8 & $2.0 \times 10^{13}$ & 1.5 \\
\hline \multirow[t]{3}{*}{ NGC 2024} & $25^{\mathrm{s}}$ & $9.1 \times 10^{6}$ & 2.9 & $>4.5 \times 10^{14}$ & \\
\hline & $40^{\mathrm{t}}$ & $2.1 \times 10^{6}$ & 2.0 & $>3.5 \times 10^{14}$ & \\
\hline & 50 & $1.6 \times 10^{6}$ & 1.7 & $>3.5 \times 10^{14}$ & \\
\hline
\end{tabular}
The critical densities for these transitions are about
Table 5. Clump parameters derived from the escape probability model.

${ }^{\mathrm{a}}$ Sievers et al. (1991), ${ }^{\mathrm{b}}$ Goldsmith \& Mao (1983), ${ }^{\mathrm{c}}$ Haschick \& Ho (1983), d Wilson et al. (1991), ${ }^{\text {e }}$ Tieftrunk et al. (1998), f Tieftrunk et al. (1995), ${ }^{\mathrm{g}}$ Jaffe et al. (1984), ${ }^{\mathrm{h}}$ Nakano \& Yoshida (1986), ${ }^{\mathrm{i}}$ Roberts et al. (1997), ${ }^{\mathrm{j}}$ McMullin et al. (1994), ${ }^{\mathrm{k}}$ Garden \& Carlstrom (1992), ${ }^{1}$ Montalban et al. (1990), ${ }^{\mathrm{m}}$ Giannakopoulou et al. (1997), ${ }^{\mathrm{n}}$ Krügel et al. (1987), ${ }^{\circ}$ Castets \& Langer (1995), ${ }^{\mathrm{p}}$ Batrla et al. (1983), ${ }^{\mathrm{q}}$ Cesaroni \& Wilson (1994), ${ }^{\mathrm{r}}$ Liseau et al. (1995), ${ }^{\mathrm{s}}$ Liseau et al. (1995), ${ }^{\mathrm{t}}$ Mezger et al. (1992). 
$6 \times 10^{7} \mathrm{~cm}^{-3}$ and $2 \times 10^{8} \mathrm{~cm}^{-3}$ respectively. From the densities in Table 5 one would conclude that these transitions are not excited. Hence, a more sophisticated model has to be applied to obtain a physically reasonable explanation of the measurements. Plume et al. (1997) suggested a two-component model or continuous density gradients to resolve this contradiction. We will discuss a self-consistent radiative transfer model including a radial density profile in the following.

\section{Cloud parameters from the nonlocal model}

\subsection{Line fitting by SimLine}

We performed non-local radiative transfer simulations using the line radiative transfer code SimLine introduced in detail in Appendix B. SimLine is a FORTRAN code to compute the profiles of molecular rotational lines in spherically symmetric clouds with arbitrary density, temperature and velocity distribution. It consists of two parts: the self-consistent solution of the balance equations for all level populations and energy densities at all radial points and the computation of the emergent line profiles observed by a telescope with finite beam width and arbitrary offset. The optical depths in the lines may vary from minus a few, corresponding to weak masking, to several thousand.

Already in the spherically symmetric description of a core we face a large number of parameters. For all quantities (hydrogen density, kinetic temperature, velocity dispersion, molecular abundances) a radial function has to be found. Regarding the limited amount of information available from the three transitions, this leaves many options open. We decided to assume simple power-law radial functions and a central region with constant parameters for all quantities in the core simulations. This reduces the number of parameters to two (central value and radial exponent) for each gas property, plus the outer and inner radius.

The parameter fit procedure used the multidimensional downhill simplex algorithm from Press et al. (1992). Although it is not the most efficient way in terms of convergence speed it turned out to be very robust in all situations considered. Because a downhill simplex code does not necessarily find the global minimum of the $\chi^{2}$ function we performed for each core several runs with randomly chosen initial simplex covering a large part of the physically reasonable parameter space. For all clouds we made at least 30 runs to get a rough idea of the topology of the $\chi^{2}$ function. For cores like W33 this turned out to be sufficient since only one large minimum showed up which was found in almost half of the runs. The other extreme is S106 where we needed almost 1000 runs to be sure that we found the global minimum. Here, the $\chi^{2}$ function was quite complex with numerous local minima. Future improvements of the fit procedure should include more sophisticated algorithms like simulated-annealing approaches.

The noise in the line profiles produces some graininess of the $\chi^{2}$ function when directly fitting the measured profiles. This results in a very slow convergence of the algorithm close to the minimum. A considerable acceleration can be obtained by not fitting the measured noisy data but a smooth approximation to them. The line profiles were represented by a superposition of a Gaussian and a Lorentzian profile. This allows a good characterisation of all measured profiles including the reproduction of asymmetric profiles, self-absorption dips and line wings.

Taking the three measured transitions and their spatial extent as the quantities to be reproduced by a $\chi^{2}$ fit we find that we are able to derive at most six parameters to a reasonable accuracy. Fits with seven or eight free parameters, although still slightly improving the numerical $\chi^{2}$ value, do not produce any significant changes above the noise limit. This is comparable to the results by Young et al. (1998) fitting the full position velocity map of a particular core in a single transition. There is however a number of additional parameters where the model can derive certain limits. Hence, we have to decide first which parameters should be fitted directly, which parameters may be constrained by preventing reasonable fits when outside a certain range, and which parameters can be guessed independently from a physical line of reasoning. This is discussed in detail for all quantities in the following subsections.

Unfortunately, it is impossible to give an comprehensive error estimate for the parameters derived from the simulations. This would need a description of the sixdimensional surface in the parameter space within which none of the observational error bars is exceeded. According to the complex topology of the $\chi^{2}$ function it is not possible to give an easy description for the six-dimensional valley around the global minimum or its boundaries.

As a simple alternative we performed only onedimensional variations to get a rough estimate of the maximum error in the parameters that we must expect. After the convergence of the $\chi^{2}$ fit, each of the fit parameters was varied up and down until one of the computed lines deviated by the assumed maximum observational error of $25 \%$ from the measured lines. The central values of the different functions were varied independently. When changing the inner radius, the central values of the gas parameters were adjusted to keep the functions in the power-law region unchanged. When varying exponents the corresponding central values were corrected in such a way that the parameters at the density of $2 \times 10^{6} \mathrm{~cm}^{-3}$ remained constant. By the selection of this density as the fix point when changing the slope, we scan about the maximum possible range of the exponents. This provides a conservative estimate of the maximum error.

\subsection{The turbulence description}

In spite of the large number of free parameters in the cloud models it is impossible to fit the line profiles with a smooth density and velocity distribution. The typical self-absorbed profiles known for all microturbulent codes 

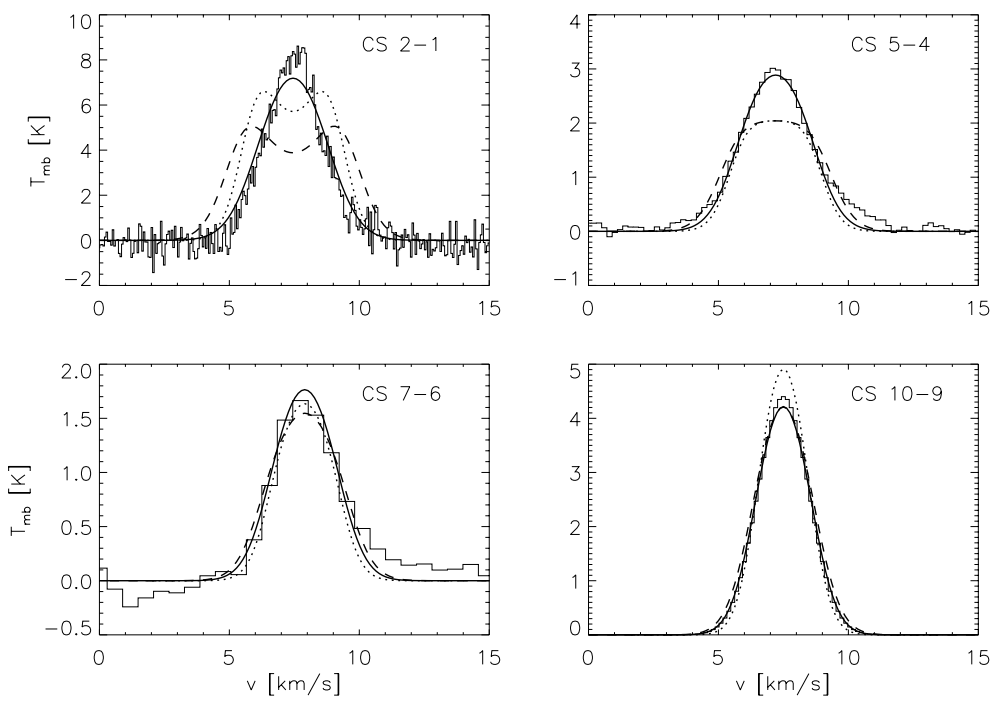

Fig. 1. Line profiles observed at the central position of S255 in four CS transitions together with best-fit models. The dashed line represents the microturbulent approximation, the dotted line stands for clumping in velocity space with cell sizes of $0.01 \mathrm{pc}$ and the solid line includes the additional effect of density clumping where the combined abundance is reduced by a factor 100 .

(White 1977) appear in this case. Thus we have to take into account the effects of internal clumping and turbulence leading to a more realistic picture and preventing strong self-absorption.

SimLine treats turbulence and clumping in a local statistical approximation following Martin et al. (1984) (see Appendix B.2). The cloud material is subdivided into small coherent units (clumps) with only thermal internal velocity dispersion. The relative motion of many units then provides the full velocity profile. Martin et al. (1984) showed that the effective optical depth of such an ensemble can be considerably reduced compared to the microturbulent approximation. The relative reduction of the total optical depth depends on the optical depth, i.e. of size the coherent units.

This description does not need any assumptions on the nature of the turbulence creating the internal cloud structure. The reduction occurs in the same way whether clumps are units of the same velocity in a medium of constant density, representing the behaviour of incompressible turbulence, or whether they are density enhancements in a thin inter-clump medium. The different nature of these two scenarios has to be taken into account, however, when computing the excitation. Both the average gas density, providing a measure for the column density and thus the line intensity, and the local density, providing the collisional coupling to the gas, enter the balance equations. In case of coherent units in velocity space both densities agree.

For density clumps we use the additional simplification to treat the cloud locally as a two-component medium neglecting the contribution of the inter-clump medium to the radiative transfer. Then the collisional excitation is provided by the density in the clumps and the column density is provided by the average density. The ratio between the two quantities reflects the filling factor of the volume occupied by dense clumps. From the mathematical point of view this is equivalent to the treatment of the cloud as a homogenous medium where the clumping occurs only in velocity space and the abundance of the radiating molecules is reduced. Hence, it is impossible to separate the influence of the filling factor from that of the molecular abundance so that the line fit provides only a combined quantity which we will denote combined abundance in the following. Beside the modification of the molecular abundance the statistical turbulence description introduces the size of the coherent units as an additional parameter.

Figure 1 demonstrates the influence of the turbulence. It shows the central line profiles of the best fits to the S255 observations using different assumptions on the turbulent nature. We selected S255 here because we can exploit the advantage of additional data for the CS 10-9 transition measured by Plume et al. (1997) providing more constraints on the cloud model ${ }^{1}$. In all models the core is optically thin in CS 7-6 and 10-9, so that the reduction of the optical depth by the turbulent clumping produces only minor changes in these lines. In CS $2-1$ the differences are, however, most obvious. In the microturbulent description and for the incompressible turbulence we find self-absorbed line profiles. A reasonable fit to the observations is only possible using the turbulence model including clumps with enhanced density. The reduction of the effective optical depth of the cloud by increasing the optical depth of the coherent units produces narrower lines providing a better fit to the observed line profiles.

We find that the parameter fits do not provide accurate values for the clump size and the combined

\footnotetext{
1 Unfortunately, Plume et al. (1997) listed only the peak intensity and FWHM so that we have to assume a Gaussian for the CS 10-9 profile here.
} 
abundances reflecting the volume filling factor. They only constrain an interval of possible values. We obtained good fits to the observations for the full range of clump sizes between about 0.005 and $0.05 \mathrm{pc}$. This size scale is confirmed by independent determinations of clump sizes by high-resolution observations in some of our cores. For NGC 2024 Mezger et al. (1992) determined a radius of dense condensations around $0.015 \mathrm{pc}$ and the interferometric studies by Wiesemeyer et al. (1997) showed values between 0.005 and 0.01 pc. In W3 Tieftrunk et al. (1998) observed compact clumps with a size of $0.02 \mathrm{pc}$ and in OMC-2 Chini et al. (1997) found dust condensations with radii between 0.01 and $0.05 \mathrm{pc}$.

For a better comparison to turbulence theory we prefer to specify the cell size in terms of the turbulent correlation length $l_{\text {corr }}$ which should be on the order of $0.1 \mathrm{pc}$ (Miesch et al. 1994; Goodman et al. 1998). The size of the units which are coherent with respect to the line radiative transfer is smaller by the ratio of the thermal line width to the total velocity dispersion. Thus the size range found corresponds to correlation lengths between 0.04 and 0.4 pc. In the following computations we use the intermediate value of $0.1 \mathrm{pc}$ as correlation length for all clouds.

Regarding the combined abundances we find two classes of objects. The majority of cores, including the example of S255, is well fitted by values between $10^{-11}$ and $10^{-10}$, whereas a second class, consisting of W3, Serpens, and S106 needs values between $10^{-10}$ and $10^{-9}$ for a good fit. Because we do not know the molecular abundances there is no way to translate these values directly into clump filling factors. Assuming the CS abundances of $1.3 \times 10^{-9}$ to $1.3 \times 10^{-8}$ obtained by Hatchell et al. (1998) for several star-forming cores, the first class corresponds to filling factors around 0.01, whereas the CS abundance of $4 \times 10^{-10}$ from Plume et al. (1997) corresponds to a filling factor of 0.1 . The filling factors in the second group are ten times higher accordingly. In all following computations we have used a combined abundance factor of $3 \times 10^{-11}$ for the first and $3 \times 10^{-10}$ for the second group. In the translation to cloud masses we will use the intermediate CS abundance of $4 \times 10^{-9}$.

As an additional parameter quantifying turbulence in a one-dimensional cloud model we have to take a radial variation of the turbulent velocity distribution into account to explain the observed size-line width and size-density relations (Larson 1969). Exponents of this radial dependence between about 0.1 and 0.7 are typically discussed (see e.g. Goodman et al. 1998). We left the width and the exponent of the turbulent velocity distribution as free parameters and obtained exponents between 0.15 and 0.65 .

\subsection{The density structure}

Any physically reasonable cloud model should include a spatial dependence of the gas parameters. Collapse simulations might provide reasonable model assumptions for the density structure. Bodenheimer \& Sweigart (1968) and Shu (1977) have shown that an isothermal sphere evolves into a power law density profile $n_{\mathrm{H}_{2}} \propto r^{-\alpha_{\mathrm{n}}}$ with $\alpha_{\mathrm{n}}=2.0$. Homologous collapse simulations provide an exponent $\alpha_{\mathrm{n}}=3.0$ (Dickel \& Auer 1994) and the free-fall collapse discussed by Welch et al. (1987) results in a density exponent $\alpha_{\mathrm{n}}=1.5$. The inside-out collapse model (Shu 1997) combined two regions of different exponents and recent more sophisticated collapse simulations (see e.g. Basu \& Mouschovias 1995) show more complex density structures with an average exponent $\alpha_{\mathrm{n}}$ between 1.5 and 1.7. Dust observations of the density profile of protostellar cores show evidence both for cores with a typical $r^{-2}$ profile and for cores with flat density structure and a sharp outer edge (André et al. 1999). Thus we expect exponents between about 1.5 and 2.0 in our power-law density model which is bound by an outer cut-off and a central constant region. Although this simple model may not reflect the whole complexity of the density profile we can hardly derive any more information from the limited observations available. We left the central density and the density exponent as free parameters to be fitted. The resulting exponents span the relatively wide range between 1.1 and 2.2 .

In the fit of the radii confining the power-law density profile we face two problems. We cannot distinguish changes of the model parameters on the smallest size scales where even the highest observed transition is thermalised because of the high density. Thus we can only set an upper limit $R_{\text {cent }}^{\max }$ to the radius of the central region where a transition from the power law behaviour in the envelope to constant parameters might occur. Only in four clouds - Serpens, Mon R2, $\rho$ Oph A, and the b-component of W49A - the strength of the CS 7-6 line sets an upper limit to the density so that we can derive the inner radius directly from the observations. In all parameter fits, we left the central radius as a free parameter. After the fit we increased the radius until the maximum deviation in one of the lines reached $5 \%$. This provides a reasonable upper limit to the inner radius in all cases where the line profiles are independent of the density structure below this limit and gives only small modifications in the four cases where the inner radius was already well constrained by the fit.

The outer radius of the cloud is also quite uncertain. We can easily provide a value for the extent of gas at densities above about $3 \times 10^{5} \mathrm{~cm}^{-3}$ based on the spatial extent of the CS 2-1 emission and the line profiles. But it is not possible to derive a reliable value for the extent of low density gas. We can only give a lower limit to the outer radius and thus the mass of the massive cores considered. As the density exponents are typically shallower than -2 , a majority of mass could be present beyond this radial limit at low densities invisible in CS. We excluded the outer radius from the parameter fit using a sufficiently large value for all clouds and performed later a separate run reducing the radius to find the minimum outer radius $R_{\text {cloud }}^{\text {min }}$ in a way equivalent to the maximisation of the inner radius described above. 
Table 6. Resulting cloud parameters for S255 assuming different exponents for the radial temperature dependence and fitting only the central line profiles.

\begin{tabular}{|c|c|c|c|c|c|c|c|}
\hline$\alpha_{\mathrm{T}}$ & $\begin{array}{l}T_{\mathrm{c}} \\
{[\mathrm{K}]}\end{array}$ & $\begin{array}{l}\langle T\rangle \\
{[\mathrm{K}]}\end{array}$ & $\alpha_{\mathrm{n}}$ & $\begin{array}{c}\left\langle n_{\mathrm{H}_{2}}\right\rangle \\
{\left[\mathrm{cm}^{-3}\right]}\end{array}$ & $\begin{array}{c}R_{\text {cloud }}^{\min } \\
{[\mathrm{pc}]}\end{array}$ & $\begin{array}{r}M_{\text {cloud }} \\
{\left[M_{\odot}\right]}\end{array}$ & $\begin{array}{c}F W H M^{\mathrm{a}} \\
{\left[{ }^{\prime}\right]}\end{array}$ \\
\hline 0.0 & 47 & 47 & -1.5 & 3300 & 1.1 & 1200 & 1.8 \\
\hline-0.12 & 73 & 52 & -1.3 & 2500 & 1.3 & 1500 & 2.2 \\
\hline-0.4 & 220 & 52 & -1.0 & 1000 & 2.5 & 7000 & 3.6 \\
\hline
\end{tabular}

${ }^{\text {a }}$ CS 2-1 transition.

\subsection{The temperature structure}

The temperature distribution of massive cores is still a matter of debate (cf. Garay \& Lizano 1999). During early phases of cloud collapse the temperature should remain constant as long as the core remains optically thin. Deviations are to be expected, however, as protostellar sources are formed in most of our cores, leading to an internal heating of the cloud. Moreover in thin outer regions external heating can be important. Based on several observational results Scoville \& Kwan (1976) set up a spherical cloud model with a warm inner region resulting in an temperature profile $T_{\text {kin }} \propto r^{\alpha_{\mathrm{T}}}$ with $\alpha_{\mathrm{T}} \approx-0.4$.

Hence, we should also derive the core temperature and the temperature exponent from the radiative transfer model. In a first run we have investigated the influence of a temperature gradient in S255 when fitting only the central line profiles. We compared the best fit models to the S255 observations using either a constant cloud temperature, a temperature decaying with the exponent -0.4 , or the temperature exponent as a free parameter. The latter case provided a best fitting exponent of -0.12 . All three fits showed an excellent agreement in terms of the central line profiles falling almost exactly on the solid curves in Fig. 1. Thus, it is impossible to favour a certain exponent from the least squares fit of the line profiles only.

In Table 6 we see the resulting values for the other core parameters. The average temperature given here is computed as the mass-weighted average up to $R_{\text {cloud }}^{\min }$. As main difference we find a kind of compensation between temperature and density exponent. The sum of both exponents is kept approximately constant to fulfil the constraints given by the line ratios. Temperature and density gradient act in a similar manner, leading to higher excitation in regions which are either denser or warmer. The variation of the density gradient, however, changes the extent of the emission in the CS 2-1 transition (the change is much smaller in the higher transitions). Consequently, we can constrain the temperature exponent when taking the observed size of the source into account. The beam convolved FWHM of the CS 2-1 emission in S255 falls between $1.5^{\prime}$ and $2.1^{\prime}$ clearly excluding models with steep temperature gradients.

In the fit procedure applied to all cores we have thus included the fit of the spatial extent. In this way we can constrain the temperature exponent but we are not able to derive exact values. In the parameter fit we always start with an isothermal cloud and change the temperature gradient in steps of 0.1 until the model provides a simultaneous good fit to the central line profiles and the spatial $F W H M$ of the emission. It turns out that the observations of all clouds except W33, W3 and Mon R2 can be fitted by an isothermal model. This indicates that a large part of the gas mass in the clouds is characterised by a uniform kinetic temperature. This isothermal behaviour, however, does not support the assumption of constant excitation temperatures like in escape probability model. The excitation temperatures show local variations which are steeper than the density gradient. In the derivation of the kinetic temperature structure we have to keep in mind, however, that we are not very sensitive to the exact value of the kinetic temperature gradient. Observations of higher transitions like CS 14-13 would be needed to obtain reliable values including full error estimates.

\subsection{Other parameters}

Beside the temperature gradient we can expect the formation of compact HII regions in the centre of a star forming core. Using the parameters of the central HII region derived by Dickel \& Auer (1994) for W49 ( $R=0.2 \mathrm{pc}$, $\left.n_{\mathrm{e}}=2.6 \times 10^{4} \mathrm{~cm}^{-3}, T_{\mathrm{e}}=10^{4} \mathrm{~K}\right)$ we have compared the resulting CS lines when either including or neglecting the HII region in the radiative transfer computations (see Appendix B.3). We find that the influence of the HII region is negligible in this example for the four CS transitions considered here.

In general HII regions have two dominant effects on the line profiles. The integrated brightness changes the molecular excitation throughout the cloud and is visible as continuum emission. Moreover, the molecular material in front of an HII region, appears partially in absorption. A large HII region with electron densities being a factor 10 or more higher than in the example above, would thus result in distinct changes in the lines. Depending on the configuration and velocity structure, the CS lines may appear in absorption or with $\mathrm{P}$ Cygni profiles. Moreover, a strong continuum emission would be observed at all frequencies considered. However, bright HII regions with high electron densities are unlikely to be that extended (Wood \& Churchwell 1989). For ultracompact HII regions the change of the line profiles by absorption is negligible due to the small angular size of the HII region so that the molecular excitation is the remaining effect. From the lack of a bright continuum underlying the lines we can, however, exclude configurations with a bright HII region here. Weaker compact or ultracompact HII regions - although possible - were not included in the fit computations as they would only influence material in their close environment which cannot be resolved in this study.

Regarding the chemical evolution of massive cores one should also expect a variation of the molecular 

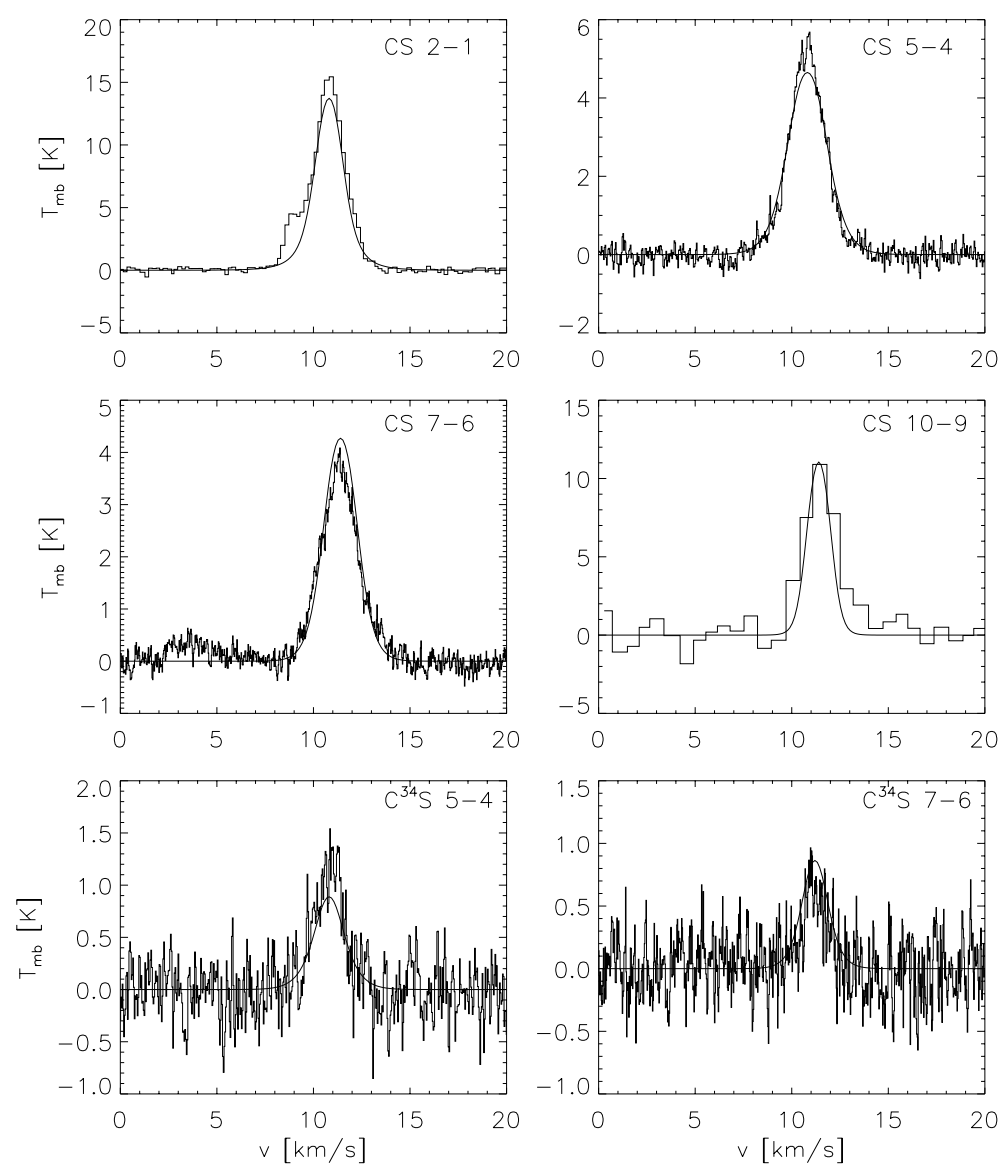

Fig. 2. Observed line profiles and best fit model to the observations of NGC 2024 using a CS to $\mathrm{C}^{34} \mathrm{~S}$ abundance ratio of 13 .

abundances of CS and $\mathrm{C}^{34} \mathrm{~S}$. However, our present knowledge is still insufficient to guess reliable values here (cf. Bergin \& Langer 1997). As discussed in Sect. 4.2, it is also not possible to fit the abundance independently from the clump filling factor, so that we adopted here a constant CS abundance of $4 \times 10^{-9}$ relative to $\mathrm{H}_{2}$ ignoring any radial variation of the abundances.

An additional test is only possible for NGC 2024 where $\mathrm{C}^{34} \mathrm{~S}$ is sufficiently bright so that we could include it in the fits. Figure 2 shows the best fit model to all six available line profiles. Equivalent to the results from the escape probability model we get the best match for a relative molecular abundance $X(\mathrm{CS}) / X\left(\mathrm{C}^{34} \mathrm{~S}\right) \approx 13$. All six lines are simultaneously fitted.

None of the cores except W49A show clearly asymmetric profiles as a signature of systematic internal velocities. The weak wings seen in a few of the other lines at low resolution are insufficient to derive any collapse or outflow model. Thus we have fitted all cores with a static model.

\subsection{Comparing the massive cores}

Table 7 lists the resulting best fit parameters for all cores with their error intervals. As discussed in Sect. 4.3 the intervals for the radii are in most cases only limited at one end. The central density refers to the value at the radius $R_{\text {cloud }}^{\min }$.
Comparing the different cores we find that the inner and outer radii are mainly determined by the selectional bias from the observability as one massive core. The most distant cores are only detectable with the KOSMA telescope if they are relatively large whereas at small distances only small cores are unresolved.

The minimum central density fitting the CS lines varies between $10^{6} \mathrm{~cm}^{-3}$ for $\mathrm{W}_{49 \mathrm{~A}^{(b)}}$ and $8 \times 10^{7} \mathrm{~cm}^{-3}$ for NGC 2024. The high value derived for NGC 2024 is due to the availability of the CS 10-9 observations tracing higher densities. With the three CS lines measured for most cores, only the density range below about $10^{7}$ can be reliably traced, so that we have to take the central density for all clouds except $\mathrm{W}_{49 \mathrm{~A}^{(b)}}$, Serpens, Mon R2, and $\rho$ Oph A as lower limits. The density exponent covers the range between -1.1 and -2.2 where the majority of clouds shows values around -1.6 corresponding to large-scale collapse models (Sect. 4.3).

Although we don't have a sample where we can expect to set up statistically significant correlations we can interpret some general relations. It turns out that the parameters are not completely independent of each other. Whereas the majority of clouds shows an average temperature between 20 and $50 \mathrm{~K}$, the three clouds which needed a larger combined abundance factor corresponding to a higher clump filling factor in the turbulence description (W3, S106, Serpens) also tend to require relatively high 
Table 7. Direct fit parameters and their likely ranges obtained in the $\chi^{2}$ minimisation.

\begin{tabular}{|c|c|c|c|c|c|c|c|c|}
\hline Sol & $\begin{array}{c}R_{\text {cent }}^{\max } \\
{[\mathrm{pc}]}\end{array}$ & $\begin{array}{c}R_{\text {cloud }}^{\min } \\
{[\mathrm{pc}]}\end{array}$ & $\begin{array}{c}n_{\text {cent }} \\
{\left[\mathrm{cm}^{-3}\right]}\end{array}$ & $\alpha_{\mathrm{n}}$ & $\begin{array}{c}T_{\text {cent }} \\
{[\mathrm{K}]}\end{array}$ & $\alpha_{\mathrm{T}}$ & $\begin{array}{c}\Delta v_{\text {cent }} \\
{\left[\mathrm{kms}^{-1}\right]}\end{array}$ & $\alpha_{\Delta v}$ \\
\hline $\mathrm{W}_{49 \mathrm{~A}^{(a)}}$ & $0.23[: 0.50]$ & $3.6[2.2:]$ & $1.2[1.0: 1.4] \times 10^{7}$ & $-1.8[1$ & $92[67: 125]$ & 0.0 & $3[5.1: 9.1]$ & 0.15 \\
\hline $\mathrm{W} 49 \mathrm{~A}^{(b)}$ & $0.92[0.59: 1.2]$ & $3.6[2.6:]$ & $9[8.5: 11.0] \times 10^{5}$ & $-1.6[1.4: 1.7]$ & 109 [85:138] & 0.0 & $7.1[5.6: 9.6]$ & .22 \\
\hline W33 & $0.27[: 0.39]$ & 1.9 [0.81:] & $9.1[7.0: 11.0] \times 10^{6}$ & $-1.9[1.1: 2.6]$ & $36[30: 42]$ & -0.2 & $4.0[2.7: 6.2]$ & .44 \\
\hline W51A & $0.57[: 0.88]$ & $3.9[2.1:]$ & $.3[4.3: 6.2] \times 10^{6}$ & $-1.6[1.2: 2.8]$ & $44[35: 53]$ & 0.0 & $8.3[6.2: 12.0]$ & 0.23 \\
\hline $\mathrm{W} 3(\mathrm{OH})$ & $0.086[: 0.16]$ & $2.2[0.54:]$ & $1.2[0.99: 1.4] \times 10^{7}$ & $-1.7[1.4: 2.6]$ & $39[32: 46]$ & 0.0 & $3.7[2.8$ & 0.08 \\
\hline W3 & $0.062[: 0.10]$ & $1.4[0.30:]$ & $6.3[4.8: 7.8] \times 10^{6}$ & $-2.0[1.7: 2.6]$ & $64[49: 79]$ & -0.2 & $3.0[2.1: 5.2]$ & 0.30 \\
\hline S255 & $0.056[: 0.077]$ & $1.1[0.66:]$ & $1.6[1.4: 1.8] \times 10^{7}$ & $-1.5[1.3: 1.7]$ & $47[41: 52]$ & 0.0 & $2.1[1.6: 2.9]$ & 0.14 \\
\hline S235B & $0.098[: 0.12]$ & $0.53[0.28:]$ & $8.3[6.8: 9.8] \times 10^{6}$ & $-2.1[1.5: 2.7]$ & $20[17: 22]$ & 0.0 & $1.7[1.3: 2.3]$ & .56 \\
\hline S106 & $0.024[: 0.038]$ & $1.2[0.13:]$ & $8.8[7.5: 9.9] \times 10^{5}$ & $-1.3[1.2: 1.4]$ & $137[103: 174]$ & 0.0 & $1.2[0.9: 1.6]$ & 0.55 \\
\hline Serpens & $0.031[0.024: 0.036]$ & $0.68[0.072:]$ & $6.4[5.6: 7.2] \times 10^{5}$ & $-1.6[1.5: 1.7]$ & $93[74: 112]$ & 0.0 & $2.8[2.2: 3.6]$ & 0.47 \\
\hline DR21 & $0.11[: 0.21]$ & $1.7[0.78:]$ & $6.5[5.3: 7.6] \times 10^{6}$ & $-1.5[1.3: 1.9]$ & $83[61: 108]$ & 0.0 & $1.8[1.4: 2.5]$ & 0.52 \\
\hline Mon R2 & $0.097[0.057: 0.12]$ & $1.3[0.33:]$ & $1.9[1.6: 2.1] \times 10^{6}$ & $-1.5[1.3: 1.8]$ & $79[61: 98]$ & -0.2 & $1.7[1.3: 2.3]$ & 0.32 \\
\hline NGC 2264 & $0.052[: 0.085]$ & $1.2[0.31:]$ & $7.0[5.9: 8.0] \times 10^{6}$ & $-1.4[1.1: 2.1]$ & $37[31: 44]$ & 0.0 & $2.9[2.2: 4.0]$ & 0.20 \\
\hline OMC-2 & $0.019[: 0.037]$ & $0.85[0.11:]$ & $1.4[1.2: 1.7] \times 10^{7}$ & $-1.5[1.1: 2.0]$ & $27[24: 31]$ & 0.0 & $1.2[0.9: 1.5]$ & 0.38 \\
\hline$\rho \mathrm{Oph} \mathrm{A}$ & $0.079[0.060: 0.10]$ & $0.68[0.16:]$ & $1.1[0.9: 1.3] \times 10^{6}$ & $-1.1[0.9: 1.3]$ & $20[15: 24]$ & 0.0 & $1.2[0.9: 1.7]$ & 0.65 \\
\hline NGC 2024 & $0.019[: 0.025]$ & $0.15[0.093:]$ & $8.0[5.8: 10.2] \times 10^{7}$ & $-2.2[1.9: 2.5]$ & $36[31: 40]$ & 0.0 & $1.2[0.8: 1.8]$ & 0.48 \\
\hline
\end{tabular}

Table 8. Resulting cloud parameters for S255 using either three or four transitions in the fit.

\begin{tabular}{|c|c|c|c|c|c|c|c|}
\hline$n_{1}$ & $\begin{array}{c}R_{\text {cent }}^{\max } \\
{[\mathrm{pc}]}\end{array}$ & $\begin{array}{c}R_{\text {cloud }}^{\min } \\
{[\mathrm{pc}]}\end{array}$ & $\begin{array}{c}n_{\text {cent }} \\
{\left[\mathrm{cm}^{-3}\right]}\end{array}$ & $\alpha_{\mathrm{n}}$ & $\begin{array}{c}T_{\text {cent }} \\
{[\mathrm{K}]}\end{array}$ & $\begin{array}{c}\left\langle n_{\mathrm{H}_{2}}\right\rangle \\
{\left[\mathrm{cm}^{-3}\right]}\end{array}$ & $\begin{array}{c}M_{\text {cloud }} \\
{\left[M_{\odot}\right]}\end{array}$ \\
\hline 4 & 0.06 & 1.1 & $1.5 \times 10^{7}$ & -1.5 & 47 & 3600 & 1000 \\
\hline 3 & 0.11 & 1.1 & $5.3 \times 10^{6}$ & -1.4 & 49 & 3700 & 1000 \\
\hline
\end{tabular}

temperatures. There is a clear correlation between the cloud temperature and the turbulent line width indicating that heating and turbulent driving might have a related cause. The cores with a significant temperature exponent also show a relatively steep density exponent whereas a steep density exponent itself does not necessarily require a temperature exponent. These internal relations should be explained from the physical nature of the clouds.

There are some peculiarities concerning four massive cores. The fit of the second component of W49A needs a large inner region with constant parameters or a very shallow decay of the density profile. Here, the main information that we get from the line profiles is the central density whereas we can hardly constrain the density exponent in the power-law range. Regarding the error bars of the parameters for Serpens we see that the the density structure of this relatively nearby cloud is well constrained by its observed size whereas the temperature structure is relatively poorly determined. In $\rho$ Oph it was not possible to fit the line profiles simultaneously with the size of the smallest core resolved in the observations. This can be explained by the clumpy structure of the whole region where the excitation of the core at the central position cannot be treated separately from the other clumps. Thus we have used for the core model a size which is six times larger than the smallest resolved clump and contains most of the strong emission observed. To fit the profiles and spatial extent of Serpens and S106 we have to assume a relatively large combined abundance (see Sect. 4.2) and a low hydrogen density in the clumps. This peculiarity could be removed when assuming some foreground CS that increases the apparent size of the massive core in CS 2-1 but does not contribute to the excitation in the core.

\section{Discussion}

\subsection{Restriction by the observed transitions}

The main constraint to the core parameters that we can derive is set by the molecule and the transitions observed. They are only sensitive to a relatively narrow density range. We can test the limitation of the fits provided by the restriction to three line profiles by comparing the results obtained for cores where we have additional CS 10-9 profiles. With the data from Plume et al. (1997) for S255 we investigate how much information is lost due to the lack of CS 10-9 data in most cores. Figure 3 shows the resulting best fits to the central line profiles in S255 when either all four lines are fitted or only the information from the lower three transitions is used. Table 8 lists the corresponding model parameters from the fits. Isothermal models provided good fits to the data and the derived physical parameters are almost identical except for the inner radius. The additional information from the CS 10-9 transition can set a smaller limit here corresponding to the higher central densities. When predicting the 10-9 line data from the best fit of the three other lines the intensity is too low by only about $20 \%$ (Fig. 3). Hence, we expect 

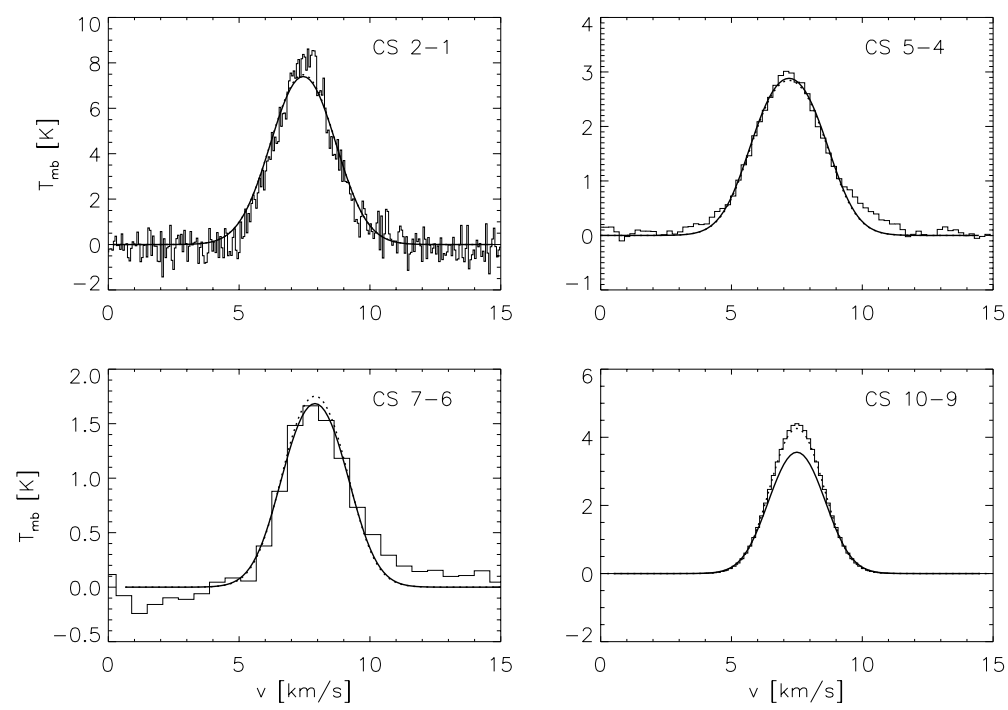

Fig. 3. Best fit models to the S255 observations fitting only the three lower transitions (solid lines) or all four transitions (dotted line).

Table 9. Error bars for the parameters in S255 using either three or four transitions in the fit.

\begin{tabular}{cccccccc}
\hline$n_{1}$ & $\begin{array}{c}R_{\text {cent }}^{\max } \\
{[\mathrm{pc}]}\end{array}$ & $\begin{array}{c}R_{\text {cloud }}^{\min } \\
{[\mathrm{pc}]}\end{array}$ & $\begin{array}{c}n_{\text {cent }} \\
{\left[\mathrm{cm}^{-3}\right]}\end{array}$ & $\alpha_{\mathrm{n}}$ & $\begin{array}{c}T_{\text {cent }} \\
{[\mathrm{K}]}\end{array}$ & $\Delta v_{\text {cent }}$ & $\begin{array}{c}\alpha_{\Delta v} \\
{\left[\mathrm{~km} \mathrm{~s}^{-1}\right]}\end{array}$ \\
\hline 4 & {$[: 0.08]$} & {$[0.66:]$} & {$[1.6: 2.2] \times 10^{7}$} & $-[1.3: 1.7]$ & {$[41: 52]$} & {$[1.6: 2.9]$} & {$[-0.01: 0.42]$} \\
3 & {$[: 0.21]$} & {$[0.68:]$} & {$[4.4: 6.1] \times 10^{6}$} & $-[1.2: 1.8]$ & {$[40: 59]$} & {$[1.7: 3.2]$} & {$[-0.07: 0.70]$} \\
\hline
\end{tabular}

Table 10. Clump parameters derived from the SimLine results. The fourth column contains the column density from the escape probability model (Table 5) for comparison and the last column the distance assumed in the mass derivation.

\begin{tabular}{lcrrrrrr}
\hline \multicolumn{1}{c}{ Source } & $\begin{array}{c}\left\langle n_{\mathrm{H}_{2}}\right\rangle \\
{\left[\mathrm{cm}^{-3}\right]}\end{array}$ & $\begin{array}{c}\left\langle N_{\mathrm{H}_{2}}\right\rangle \\
{\left[10^{23}\right.}\end{array}$ & $\begin{array}{c}N_{\mathrm{H}_{2}}(\mathrm{EP}) \\
\left.\mathrm{cm}^{-2}\right]\end{array}$ & $\begin{array}{r}\langle T\rangle \\
{[\mathrm{K}]}\end{array}$ & $\begin{array}{c}M_{\text {cloud }} \\
{\left[M_{\odot}\right]}\end{array}$ & $\begin{array}{r}M_{\text {vir }} \\
{\left[M_{\odot}\right]}\end{array}$ & $\begin{array}{c}D \\
{[\mathrm{pc}]}\end{array}$ \\
\hline W49A $^{(a)}$ & $1.4 \times 10^{3}$ & 2.6 & 4.6 & 92 & 15000 & 22000 & 11000 \\
W49A $^{(b)}$ & $1.7 \times 10^{3}$ & 0.80 & 4.8 & 109 & 19000 & 14000 & 11000 \\
W33 & $4.6 \times 10^{3}$ & 2.3 & 3.8 & 28 & 7400 & 6300 & 4000 \\
W51A & $3.9 \times 10^{3}$ & 3.0 & 9.8 & 43 & 54000 & 30000 & 7500 \\
W3(OH $)$ & $7.7 \times 10^{2}$ & 1.0 & 1.5 & 39 & 1900 & 1900 & 2200 \\
W3 & $2.7 \times 10^{3}$ & 3.6 & 9.7 & 41 & 1700 & 1100 & 2200 \\
S255 & $2.8 \times 10^{3}$ & 1.1 & 0.78 & 47 & 870 & 530 & 2500 \\
S235B & $4.5 \times 10^{3}$ & 0.66 & 0.22 & 20 & 180 & 210 & 1800 \\
S106 & $7.2 \times 10^{2}$ & 0.32 & 0.23 & 137 & 280 & 200 & 600 \\
Serpens & $7.3 \times 10^{2}$ & 0.22 & 0.17 & 93 & 53 & 80 & 310 \\
DR21 & $1.6 \times 10^{3}$ & 0.82 & 0.79 & 83 & 1800 & 1800 & 3000 \\
Mon R2 & $6.1 \times 10^{2}$ & 0.22 & 0.18 & 54 & 310 & 260 & 950 \\
NGC 2264 & $1.1 \times 10^{3}$ & 0.46 & 0.71 & 37 & 450 & 720 & 800 \\
OMC-2 & $6.6 \times 10^{2}$ & 0.34 & 0.24 & 27 & 95 & 52 & 400 \\
$\rho$ Oph A & $1.3 \times 10^{3}$ & 0.12 & 0.12 & 20 & 96 & 34 & 160 \\
NGC 2024 & $2.2 \times 10^{4}$ & 1.3 & 1.6 & 36 & 17 & 35 & 420 \\
\hline
\end{tabular}

reliable results also for those cores where only three lines are observed but it would be favourable to add information from higher transitions for a better resolution of the densest inner region.

Moreover, a fourth line will reduce the error bars of the parameters. In Table 9 we compare the error obtained for the S255 observations using either the three- or the four-lines fit. As discussed above the possible range of the inner and outer radii is limited only in one direction. Thus, the fourth line mainly reduces the uncertainty of the inner radius. It hardly influences the error of the outer radius and the density at the inner radius but it also reduces the 
error of the density exponent, the temperature and the velocity structure. Thus the inclusion of additional lines in the model fits would also give a better constraint of the parameters derived.

\subsection{Comparison of the methods}

Table 10 shows quantities characterising the global properties of the clouds computed from the fit parameters in Table 7 . The average density in the second column is given by the cloud mass within the outer radius. We see the strong discrepancy between the average density (Table 10) and the central clump density (Table 7 ) reflecting a very inhomogenous structure with low volume filling factor of dense clumps.

In the Cols. 3 and 4 of Table 10 one can compare the average column density towards the centre in the nonlocal model with the column density computed from the escape probability model. Here, we have used the molecular column densities from Table 5 assuming a CS abundance of $4 \times 10^{-9}$. We find an agreement within a factor of about 1.5 , despite the completely different analysis applied, except for W49A, W51, and W3 where the column density from the escape probability model is more than a factor of two higher and S235B where it is lower. S235B, W49A, and W3 are the smallest sources in our sample unresolved even in the CS 2-1 beam. Here, the beam filling factors used in the escape probability model are uncertain so that they may be responsible for the difference. For W51A, no simple explanation for the difference is obvious. It is however, by far the most massive core in our sample so that it might be somewhat peculiar from that point of view. In general, we find that the escape probability analysis provides a reasonable determination for the column density when we have a good estimate of the beam filling factor. It fails to derive correct densities or sizes.

\subsection{Core masses}

Columns 6 and 7 in Table 10 shows the core masses computed in two different ways. Column 6 gives the integrated mass of the model cloud assuming the smallest fitting outer radius and the maximum possible central radius. The influence of the inner radius on the total mass is negligible, but the uncertainty from the lack of information on the outer radius has to be kept in mind. Increasing the amount of virtually invisible material around the core by increasing the outer cloud radius can easily increase the total mass by more than a factor 10 . As the mass computation relies on the knowledge of the CS molecular abundance (Sect. 4.2), the resulting values are to be changed if the true abundances deviate from the assumed value of $4 \times 10^{-9}$.

Column 7 contains the core virial mass assuming equipartition of kinetic and gravitational energy in a homogenous spherical cloud. We used the central CS 21 line profile and the size of the cloud visible in this transition to estimate the velocity dispersion in the line of sight and the radius. Following Lang (1980) we obtain the virial mass by

$M_{\mathrm{vir}}=0.0183 \Delta v^{2} D \sqrt{\Delta \alpha \Delta \delta}$

where $\Delta v$ is the $F W H M$ of the line (Table 2), D the distance of the source, and the $\Delta \alpha$ and $\Delta \delta$ the FWHM of the source in declination and rectascension in arcmin from Table 4.

For all clouds except $\rho$ Oph A the agreement between the two masses falls within a factor of two. The behaviour of $\rho \mathrm{Oph} \mathrm{A}$ is due to the radius of the excitation model which is larger than the smallest resolved core, as discussed above. Hence, it provides a larger mass than the virial estimate which uses this visible core size. The general good agreement is quite amazing regarding the uncertainty of the outer boundary of the models. The clouds seem to be virialised and the CS abundance estimate holds approximately for all clouds.

The agreement of the mass from the SimLine fits with the virial mass and independent estimates from the literature indicates that the cores are well confined and our minimum outer radius corresponds to a real, relatively sharp boundary for most cores in agreement with the results from continuum observations of several cores by André et al. (1999). Future investigations are, however, necessary to confirm this result because the nature of virialisation is still not understood and it is therefore not clear how much of the "invisible" low density mass would contribute to the virial mass.

\subsection{Comparison with other observations}

To judge the reliability of the parameters derived here, we can compare them with core parameters obtained independently from observations in other tracers and with other telescopes. In general they provide only values for few of the cloud parameters but they may serve as an independent test of our results. We cannot include a complete discussion of the literature concerning the 15 massive cores considered here. Rather we restrict ourselves to a few selected observations showing the general power and limitations of the method.

For NGC 2024 we have compared our data with results from complementary high-resolution observations in Table 11. Mezger et al. (1992) combined the results of SEST observations at $1.3 \mathrm{~mm}$ with IRAM $30 \mathrm{~m}$ continuum maps at $870 \mu \mathrm{m}$ to identify several clumps in NGC 2024 and to deduce their physical properties from the continuum fluxes. The given values correspond to FIR5 falling at our central position. Wiesemeyer et al. (1997) used a spherically symmetric continuum transfer model to derive the physical parameters of FIR 5 from $3 \mathrm{~mm}$ continuum observations taken with the IRAM Plateau de Bure interferometer combined with the data from Mezger et al. (1992) and VLA $1.3 \mathrm{~cm}$ observations of Gaume et al. (1992). Depending on the assumed luminosity and dust properties they found a range of parameters fitting the observed 
Table 11. Comparison of the NGC 2024 FIR5 parameters with results from investigations based on other high resolution observations.

\begin{tabular}{lrrrrr}
\hline & $R$ & $\begin{array}{c}n_{\text {cent }} \\
{\left[\begin{array}{c}10^{8} \\
\mathrm{~cm}^{-3}\end{array}\right]}\end{array}$ & $\begin{array}{c}N_{\mathrm{H}_{2}, \text { cent }} \\
{\left[\begin{array}{c}10^{24} \\
\left.\mathrm{~cm}^{-2}\right]\end{array}\right.}\end{array}$ & $T$ & $M_{\text {cloud }}$ \\
& {$[\mathrm{pc}]$} & {$\left[M_{\odot}\right]$} \\
\hline Mezger $^{\mathrm{a}}$ & 0.007 & 4.8 & 22 & 19 & 20 \\
Wiesemeyer $^{\mathrm{b}}$ & $\approx 0.01$ & $0.9-2.3$ & $3.1-8.4$ & $16-19$ & $16-50$ \\
this paper $^{\mathrm{c}}$ & 0.15 & $>0.8$ & 9.9 & 36 & 17 \\
\hline
\end{tabular}

a IRAM $30 \mathrm{~m} 870 \mu \mathrm{m}$, SEST $1.3 \mathrm{~mm}$ continuum, Mezger et al. (1992).

${ }^{\mathrm{b}}$ IRAM PdB 3 mm, IRAM $30 \mathrm{~m} 870 \mu \mathrm{m}$, VLA $1.3 \mathrm{~cm}$ continuum, Wiesemeyer et al. (1997).

${ }^{c}$ KOSMA CS and $\mathrm{C}^{34} \mathrm{~S}$, IRAM $30 \mathrm{~m}$ CS as discussed in the text.

continuum. The last line in Table 11 represents the results from the SimLine fit to our data. In contrast to Table 10, the central column density given here is not averaged but computed towards a central clump to allow a better comparison with the dust observations which are able to resolve this clump.

We see that one cannot reveal the true radius of the core from our low resolution observations. Moreover, the CS observations cannot trace the same high densities as the dust observations so that they provide only a lower limit. It is, however, already close to the central density given by Wiesemeyer et al. (1997). The mass and column density derived from our radiative transfer computations agree quite well with the values provided by the high-resolution observations. A possible explanation for the difference between the gas kinetic temperature and the dust temperatures was provided already by Schulz et al. (1991). They performed $\mathrm{NH}_{3}$ and $\mathrm{CS}$ observations of NGC 2024 and obtained temperatures between 35 and $40 \mathrm{~K}$ at the position considered. Using a two-component dust model they demonstrated that these temperatures are also consistent with the observations by Mezger et al. (1992). Altogether we are able to derive realistic values for the core parameters with a clumpy radiative transfer model even if we are not able to deduce the exact object size as we cannot resolve it.

W51 consists of three compact molecular cores located within about one arcmin. Interferometric observations by Young et al. (1998) and the combination of line and continuum measurements by Rudolph et al. (1990) seem to indicate collapse of the component W51e 2 with a mass of about $40000 M_{\odot}$. The mass determined by the SimLine fit is $54000 M_{\odot}$. The FCRAO and KOSMA observations show no signatures of collapse as they are probably blurred by our low spatial resolution. Sievers et al. (1991) obtained temperatures between $20 \mathrm{~K}$ and $57 \mathrm{~K}$ and Zang \& Ho (1997) derived 40-50 K for an inner region of about $0.2 \mathrm{pc}$ and $25-30 \mathrm{~K}$ for the outer cloud based on $\mathrm{NH}_{3}$ observations. We were able to fit the observations with an isothermal cloud at $44 \mathrm{~K}$, however cannot exclude such a temperature structure. Young et al. (1998) used an LTE code assuming spherical or spheroidal symmetry to simulate the inner $0.2 \mathrm{pc}$ region of W51e2 fitting the observed ammonia data. They obtained density gradients of -1.8 to -2.2 somewhat steeper than in our fit, indicating that a dense central region might be surrounded by an envelope with a flatter density gradient. Their central densities between 1.5 and $22 \times 10^{6} \mathrm{~cm}^{-3}$ bracket our value of $5 \times 10^{6}$. The assumption of a smooth medium by Young et al. (1998) results in a central temperature estimate below $25 \mathrm{~K}$ and a steep temperature gradient. I.e. significantly lower temperatures than in our clumpy turbulent model. We have tested this behaviour by trying to fit the data without clumping in our model and also got low temperatures below $20 \mathrm{~K}$ but quite bad $\chi^{2}$ values. Thus the correct treatment of the internal clumping is essential for a reliable temperature derivation.

W3 $(\mathrm{OH})$ was studied e.g. by Wilson et al. (1991) using VLA observations of methanol and $\mathrm{OH}$ and by Tieftrunk et al. (1998) and Helmich et al. (1996) with single dish observations of ammonia and HDO, respectively. The KOSMA beam covers several maser spots and a luminous mm continuum source - probably a class 0 object. Wilson et al. (1991) obtained a kinetic temperature of the molecular cores of $20 \mathrm{~K}$, whereas Tieftrunk et al. (1998) derived $27 \mathrm{~K}$. Both agreed with our total mass estimate of about $2000 M_{\odot}$. The radius of $1.3 \mathrm{pc}$ determined by Tieftrunk is somewhat smaller than the value computed from our model, but clearly within the error bar. From the HDO excitation Helmich et al. (1996) conclude dust temperatures above $100 \mathrm{~K}$ at densities between $10^{5}$ and $10^{6} \mathrm{~cm}^{-3}$, with few embedded clumps at $10^{7} \mathrm{~cm}^{-3}$. This clump density and the column density of about $2 \times 10^{23} \mathrm{~cm}^{-2}$ agree approximately with the values from our spherical model. The temperature of $39 \mathrm{~K}$ determined from the CS observations falls into the range discussed but the difference to the methanol and ammonia values asks for an explanation. Around the massive core there is probably still an extended envelope of gas at low densities insufficient to excite the observed CS transitions (Tieftrunk et al. 1998).

W3 was studied in detail by Tieftrunk et al. (1995, 1997, 1998) using $\mathrm{C}^{34} \mathrm{~S}, \mathrm{C}^{18} \mathrm{O}, \mathrm{NH}_{3}$, and continuum observations. Our beam covers the two bright components W3 Main and W3 West. Moreover, the region contains some ultracompact HII regions related to infrared sources. The molecular line emission peaks at a position close to W3 West. The line velocity of our CS observations at $-42 \mathrm{kms}^{-1}$ agrees with the velocity of W3 West indicating this component as the main originator of the observed CS emission. The combination of single dish and VLA observations by Tieftrunk et al. $(1997,1998)$ showed extended gas at a temperature of $25-45 \mathrm{~K}$, a density of $10^{4} \mathrm{~cm}^{-3}$, an size of about $1 \mathrm{pc}$, and a total mass of 1100-1400 $M_{\odot}$. This corresponds well to the parameters derived from the KOSMA observations. We have traced the emission to the somewhat larger radius of $1.4 \mathrm{pc}$, but within a radius of $1 \mathrm{pc}$ we get about the same average density of $0.7 \times 10^{4} \mathrm{~cm}^{-3}$. Our mass estimate of $1100 M_{\odot}$ 
and the average temperature of $41 \mathrm{~K}$ also agree. The VLA observations showed several very compact clumps with a size of $0.02 \mathrm{pc}$, densities of $10^{7} \mathrm{~cm}^{-3}$, and $T_{\text {kin }}=250 \mathrm{~K}$. They are not resolvable from our data, but correspond to the clumps in the turbulence description and the core parameters derived from the radiative transfer model show a similar size and density. In the smooth temperature distribution assumed in the radiative transfer model we are not able to resolve hot spots with $250 \mathrm{~K}$ but found the need for an increased temperature towards the centre.

From the group of low mass cores, Castets \& Langer (1995) analysed CS observations of OMC-2 by means of an LVG analysis providing $T_{\text {kin }}=24 \mathrm{~K}$, a density of $9 \times 10^{5} \mathrm{~cm}^{-3}$ and a CS column density of $5.4 \times 10^{13} \mathrm{~cm}^{-2}$ in agreement with our results. They found already indications for substructure with clump radii of about $0.022 \mathrm{pc}$ and higher densities in observations with higher resolution. Our analysis shows densities of at least $1.4 \times 10^{7} \mathrm{~cm}^{-3}$ at a scale of 0.019 pc. The virial mass of $71 M_{\odot}$ computed by Castets \& Langer is only somewhat smaller than our mass estimate of $95 M_{\odot}$. Recent $1.3 \mathrm{~mm}$ observations by Chini et al. (1997) show at least 11 embedded condensations in OMC-2 with masses between 5 and $8 M_{\odot}$ and temperatures between 20 and $33 \mathrm{~K}$ whereas $350 \mu \mathrm{m}$ continuum data by Lis et al. (1998) reveal even 30 clumps but lower temperatures of $17 \mathrm{~K}$ supporting our approach of the clumpy cloud model.

The comparison shows that different tracers see different parts of a cloud corresponding to different physical conditions. Results from other authors based on CS observations agree in most cases quite well, whereas the results from other tracers may considerably differ. The relatively large uncertainty in the temperature structure that we cannot resolve within our analysis asks for additional observations in higher transitions or at better spatial resolution. For nearby clouds like NGC 2024, OMC-2 or $\rho$ Oph A we get a good agreement with results from high-resolution or even interferometric observations, whereas for distant massive cores like W49A and W51A there are several open question, especially regarding the temperature structure.

\subsection{The physical nature of massive cores}

All massive cores seem to be approximately virialised independent of their internal structure with respect to the number, distribution and luminosity of young stars. Although one could expect that violent bipolar outflows observed in some cores will drastically change the energy balance in the core, the physics of the turbulence in the cores seems to be extremely stable guaranteeing a continuous state of virialisation.

The relatively sharp outer boundary suggested by the mass estimates can be interpreted in terms of collapse models. The collapse of an isothermal sphere would result in a self-similar density distribution without clear boundary whereas our results rather tend towards the scenario of a finite-size Bonnor-Ebert condensation (Bonnor 1956).
The outer boundary is, however, not well determined but only set by the mass constraints because the radiative transfer model itself cannot exclude a continuation of the density structure to larger radii.

The density exponent of about -1.6 derived for most cores is consistent with several collapse models (see Sect. 4.3) but deviations from the exponent for particular clouds up to values around -2 have to be explained.

On the other hand we have seen that simple collapse models are not relevant for the massive cores considered here. Clumpiness is a main feature of all clouds and smooth microturbulent models are not able to explain the observed lines. In agreement with other high-resolution observations we find typical clump sizes of $0.01-0.02 \mathrm{pc}$ at least for the nearby cores. In massive distant cores the situation might be more complex including a hierarchy of clump sizes resulting in a larger uncertainty of the temperature profile derived from our model.

\section{Conclusions}

We have shown that the careful analysis of multi-line single dish observations with a relatively large beam can provide a set of information comparable to single-line interferometric observations. From a careful excitation analysis using a self-consistent radiative transfer computation it is possible to deduce some sub-resolution information. We can infer clump sizes, masses and densities at scales below a tenth of the beam size. However, interferometric observations are necessary to determine the exact core geometry including the location and number of clumps within a dense core.

The spherically symmetric radiative transfer code used here is able to take into account radial gradients in all quantities and internal clumpiness of the cloud. It enables a reliable deduction of the physical parameters from line profiles observed in sources with a size close to the spatial resolution limit. The method allows to analyse similar observations of objects like star-forming cores in distant galaxies unresolvable by all today's means. For a better resolution of the internal temperature structure the approach should be combined with sophisticated models on the energy balance including the continuum radiative transfer in the future. Although the simple escape probability analysis gives a reasonable estimate for the column density, it fails regarding the density and temperature structure.

The line analysis shows two essential points:

i) The main constraints on the structural quantities which can be deduced from the observations are set by the tracer. The range of densities and temperatures that one can determine from the radiative transfer calculations is restricted by the transitions observed. In case of the CS $2-1,5-4$, and $7-6$ lines, the covered densities range from about $2 \times 10^{5}$ to $10^{7} \mathrm{~cm}^{-3}$. With additional information from the CS 10-9 the upper limit can be extended by another factor 5 . The information from the rarer $\mathrm{C}^{34} \mathrm{~S}$ isotope cannot extend the density interval but reduces the 
error bars and provides better estimates for the clumpiness of the medium. The high resolution observations discussed in Sects. 3.2 and 5.4 show that different tracers provide access to different types of information whereas the parameters from our CS observations agree well with the CS results there.

ii) Temperature and clumpiness are related quantities. When turbulent clumping in the cloud is neglected, the temperature determination will necessarily fail. On the other hand does accurate information on the clumpy structure of a massive core help to constrain the temperature structure. Additional observations in higher transitions or complementary estimates of the clumpiness will help to reduce the uncertainty of the temperatures. Thus spatial resolution is still essential. For nearby clouds we get a good agreement with results from other high-resolution observations, but for distant massive cores the temperature structure is still an open question.

All massive cores that we have analysed are characterised by turbulent clumpiness with typical clump sizes of $0.01-0.02$ pc. The clouds are approximately virialised and show density gradients around -1.6 but with a scatter between -1.1 and -2.2 . Large parts of the cores follow a constant temperature, but we must admit a considerable uncertainty in the most inner and outer parts. The correlation between the cloud temperature and the turbulent line width indicates that related processes should be responsible for heating and turbulent driving.

Future observations of dense cores should focus on different tracers to gain access to additional information which cannot be deduced from a single tracer such as CS. As a drawback, the full uncertainty of today's chemical models will enter and partially limit the interpretation of the observations.

Acknowledgements. We thank J. Howe for providing us with the CS 2-1 observational data. We are grateful to the anonymous referee for many detailed comments helping to improve the paper considerably. This project was supported by the Deutsche Forschungsgemeinschaft through the grant SFB 301C. The KOSMA 3 m radio telescope at Gornergrat-Süd Observatory is operated by the University of Cologne and supported by the Land Nordrhein-Westfalen. The receiver development was partly funded by the Bundesminister für Forschung and Technologie. The Observatory is administered by the Internationale Stiftung Hochalpine Forschungsstationen Jungfraujoch und Gornergrat, Bern. The research has made use of NASA's Astrophysics Data System Abstract Service.

\section{Appendix B: SimLine - A one-dimensional radiative transfer code}

\section{B.1. The radiative transfer problem}

SimLine solves the line radiative transfer problem discussed in Appendix A.1 in a spherically symmetric configuration by means of a $\lambda$-iteration. The code is similar to the concept described by Dickel \& Auer (1994) but it contains several extensions and achieves a higher accuracy from an adaptive discretisation of all independent quantities.

SimLine integrates the radiative transfer Eq. (A.2) for a number of rays numerically. In spherical symmetry it is sufficient to consider the propagation of radiation in one arbitrary direction which is taken as $z$ here. The integral is computed stepwise from $z_{i-1}$ to $z_{i}$

$$
\begin{aligned}
I_{\nu}\left(p, z_{i}\right) & =\exp \left(-\int_{z_{i-1}}^{z_{i}} \mathrm{~d} z \kappa_{\nu}(p, z)\right)\left[I_{z}\left(\nu, p, z_{i-1}\right)\right. \\
& \left.+\int_{z_{i-1}}^{z_{i}} \mathrm{~d} z \epsilon_{\nu}(p, z) \exp \left(\int_{z_{i-1}}^{z} \mathrm{~d} z^{\prime} \kappa_{\nu}\left(p, z^{\prime}\right)\right)\right]
\end{aligned}
$$

where $p$ denotes the displacement variable perpendicular to the $z$ direction. To minimise the discretisation error the integral does not use the source function but only the emission and absorption coefficients which are linear in the level populations. They are assumed to change linearly between the grid points and the exact integration formula for a linear behaviour (which is not given here, but can be derived straight forward) is applied. This approach provides a reduction of the integration error to third order. The radial grid is dynamically adjusted to give a maximum variation of the level populations between two neighbouring points below a certain limit. In case of strong velocity gradients additional points are included on the $z$-scale for a sufficiently dense sampling of changes in the profile function. The incident radiation at the outer boundary of the cloud is assumed to follow a black body spectrum.

In spherical symmetry the spatial integration of the radiative energy density (Eq. (A.1)) can be reduced to

$$
\begin{aligned}
& u(r)=\frac{2 \pi}{r c} \int_{-r}^{r} \mathrm{~d} z^{\prime} \int_{-\infty}^{\infty} \mathrm{d} \nu \Phi_{\nu}\left(p^{\prime}, z^{\prime}\right) I_{\nu}\left(p^{\prime}, z^{\prime}\right) \\
& \text { with } \quad p^{\prime}=\sqrt{r^{2}-z^{\prime 2}} .
\end{aligned}
$$

The grid of rays tangential to the radial grid is refined by additional rays at intermediate $p^{\prime}$ values to guarantee a sufficiently dense sampling on the $z^{\prime}$ scale. The integration uses a cubic spline interpolation.

With the values of the radiative energy density at each radial point and for each transition, the system of balance equations can be solved providing new level populations. Here, a LU decomposition algorithm with iterative improvement (Press et al. 1992) is used. The new level populations are used in the next iteration as input for the radiative transfer equation. The whole $\lambda$-iteration scheme is solved using the convergence accelerator introduced by Auer (1987).

Depending on the physical situation the initial guess is either the optically thin limit, thermalisation or the solution of the radiative transfer equation using the LVG approximation (Eq. (A.4)). The stability of the local radiation field is used as convergence criterion. The number of iterations required for convergence depends strongly on the optical depth of the model cloud. For the examples discussed in this paper only about a dozen iterations were necessary but other test cases with complex molecules like 
water, non-monotonic velocity gradients, and high optical depths require several hundred iterations.

\section{B.2. The local turbulence approximation}

The turbulence description uses two additional parameters for each spatial point: the width of the velocity distribution $\sigma$ providing the local emission profile for optically thin lines and the correlation length of the macroturbulent density or velocity distribution $l_{\text {corr }}$.

The width of the velocity distribution $\sigma$ is composed of a turbulent and a thermal contribution

$\sigma=\frac{\nu_{0}}{c} \sqrt{\frac{2 k T_{\text {kin }}}{m_{\text {mol }}}+\frac{2}{3}\left\langle v_{\text {turb }}^{2}\right\rangle}$

where a Maxwellian distribution of turbulent velocities is assumed. The relation between the FWHM and the variance of the turbulent velocity distribution is given by $\left.F W H M\left(v_{\text {turb }}\right)=\sqrt{8 / 3 \times \ln 2\left\langle v_{\text {turb }}^{2}\right\rangle}\right)$. The long range variation of the turbulence spectrum as described by means of a Kolmogorov or Larson exponent is simulated by a radially varying turbulent velocity dispersion $\sqrt{\left\langle v_{\text {turb }}^{2}\right\rangle} \propto r^{\gamma}$. Exponents $\gamma$ between about 0.1 (Goodman et al. 1998) and 0.7 (Fuller \& Myers 1992) are observationally justified.

For the local treatment of coherent units in a turbulent medium the considered volume element is subdivided into numerous clumps with a thermal internal velocity dispersion. When each clump is characterized by a Gaussian density distribution of molecules with about the same velocity $n(r)=n_{0} \times \exp \left(-r^{2} / r_{\mathrm{cl}}^{2}\right)$ the effective absorption coefficient at the considered velocity for the whole medium is

$\kappa_{\mathrm{eff}}=n_{\mathrm{cl}} \times \pi r_{\mathrm{cl}}^{2} \int_{0}^{\tau_{\mathrm{cl}}} \frac{1-\exp (-\tau)}{\tau} \mathrm{d} \tau$

where $n_{\mathrm{cl}}$ is the number density of contributing cells and $\tau_{\mathrm{cl}}=\sqrt{\pi} \kappa r_{\mathrm{cl}}$ is their central opacity (Martin et al. 1984). As the clumps size $r_{\mathrm{cl}}$ is the length on which the abundance of molecules within the same thermal velocity profile is reduced by the factor $1 / \mathrm{e}$, we can compute it from the correlation length of the velocity or density structure by $r_{\mathrm{cl}}=l_{\text {corr }} \times \sigma_{\mathrm{th}} / \sigma$.

When the turbulent velocity dispersion $\sigma$ is at least three times as large as the thermal velocity dispersion $\sigma_{\mathrm{th}}$, we obtain an effective absorption coefficient

$\kappa_{\mathrm{eff}, \nu}=n_{\mathrm{ges}} \pi r_{\mathrm{cl}}^{2} \times A\left(\tau_{\mathrm{cl}}\right) \times \frac{\sigma_{\mathrm{th}}}{\sigma} \exp \left(-\frac{\left(\nu-\nu_{0}\right)^{2}}{\sigma^{2}}\right)$

with

$A(\tau)=\frac{1}{\sqrt{\pi}} \int_{-\infty}^{\infty} \mathrm{d} v \int_{0}^{\tau \exp \left(-v^{2}\right)} \frac{1-\exp \left(-\tau^{\prime}\right)}{\tau^{\prime}} \mathrm{d} \tau^{\prime}$.

Here, $n_{\text {ges }}$ is the total number density of clumps. In case of incompressible turbulence, i.e. clumping in velocity space, it is equal to the reciprocal cell volume. For small values of the clump opacity, $A\left(\tau_{\mathrm{cl}}\right)$ is identical to $\tau_{\mathrm{cl}}$ and we reproduce the microturbulent limit. For large $\tau_{\mathrm{cl}}$, the function $A\left(\tau_{\mathrm{cl}}\right)$ saturates and we obtain a significant reduction of the effective absorption coefficient. In case of density clumps, $\kappa_{\text {eff }}(\nu)$ is further reduced by the filling factor entering $n_{\text {ges }}$. In SimLine, this is simulated by a corresponding artificial reduction of the molecular abundance.

\section{B.3. The central HII region}

To simulate the effect of a central continuum source in the cloud, it is possible to assume an HII region in the cloud centre. The HII region is characterised by two parameters, the electron density $n_{\mathrm{e}}$ and the kinetic electron temperature $T_{\mathrm{e}}$.

The absorption coefficient for electron-ion bremsstrahlung in the Rayleigh-Jeans approximation is given by:

$\kappa_{\nu}=\frac{8}{3 \sqrt{2 \pi}} \frac{e^{6}}{\left(4 \pi \epsilon_{0} m_{\mathrm{e}}\right)^{3} c}\left(\frac{n_{\mathrm{e}}}{\nu}\right)^{2}\left(\frac{m_{\mathrm{e}}}{k T_{\mathrm{e}}}\right)^{3 / 2} \ln \Lambda$

where it is assumed that the gas is singly ionised and $\Lambda$ is given by

$\Lambda=\left(\frac{2 k T_{\mathrm{e}}}{\gamma m_{\mathrm{e}}}\right)^{3 / 2} \frac{4 \pi \epsilon_{0} m_{\mathrm{e}}}{\pi \gamma e^{2} \nu} \approx 4.96 \times 10^{7}\left(\frac{T}{\mathrm{~K}}\right)^{3 / 2} \frac{\mathrm{Hz}}{\nu}$

for $T_{\mathrm{e}}<3.6 \times 10^{5} \mathrm{~K}$. The quantities $e$ and $m_{\mathrm{e}}$ denote the electron charge and mass, $c$ is the vacuum light velocity, and $\gamma=1.781$ (Lang 1980).

For a thermal plasma, the emission coefficient follows from the Planck function

$\epsilon_{\nu}=\kappa_{\nu} \times B_{\nu}\left(T_{\mathrm{e}}\right)$

In the radiative transfer computations the frequency dependence of these continuum coefficients is neglected within the molecular line width. Within the HII region, we substitute the molecular coefficients in Eq. (B.1) by the quantities from Eqs. (B.7) and (B.9) so that we locally switch to a continuum radiative transfer.

\section{B.4. Computation of beam temperatures}

When the level populations are known, the beam temperature relative to the background is computed by the convolution of the emergent intensity with the telescope beam.

$T_{\mathrm{mb}}=\frac{c^{2}}{2 k \nu_{0}^{2}} \frac{\int_{0}^{2 \pi} \mathrm{d} \phi \int_{0}^{\infty} \mathrm{d} p p\left(I_{\nu}^{\mathrm{S}}(p)-I_{\mathrm{bg}}\right) f_{\mathrm{mb}}(p, \phi)}{\int_{0}^{2 \pi} \mathrm{d} \phi \int_{0}^{\infty} \mathrm{d} p p f_{\mathrm{mb}}(p, \phi)}$.

The intensity $I_{\nu}^{\mathrm{S}}(p)$ is the value on the cloud surface $I_{\nu}^{\mathrm{S}}(p)=I_{\nu}\left(p, \sqrt{R_{\text {cloud }}^{2}-p^{2}}\right)$. We assume a Gaussian profile for the telescope beam

$f_{\mathrm{mb}}(p, \phi)=\exp \left(\frac{-\left(p-p_{\text {offset }}\right)^{2}\left(1+\phi^{2}\right)^{2}}{\sigma_{\mathrm{mb}}^{2}}\right)$. 
The projected beam width is computed from the angular width by $\sigma_{\mathrm{mb}}=\pi / 648000 D \sigma_{\mathrm{mb}}{ }^{[\prime]}=2.912 \times$ $10^{-6} D F W H M\left[^{[\prime}\right]$ where $D$ is the distance of the cloud. The program computes a radial map with arbitrary spacings.

\section{B.5. The general code design}

The design of the code is directed towards a high accuracy of the computed line profiles. All errors in the different steps of the program are explicitly user controlled by setting thresholds. All discretisations necessary to treat the problem numerically are performed in an adaptive way, i.e. there is no predefined grid and all grid parameters will change during the iteration procedure. The system of balance equations is truncated whenever the excitation of all higher levels falls below a chosen accuracy limit.

Furthermore, the code was pushed towards a high flexibility, i.e. the ability to treat a very broad range of physical parameters with the same accuracy and without numerical limitations. The systematic velocities e.g. may range from 0 to several times the turbulent velocity and the optical depths may vary from negative values for weak masing to values of several thousands.

The program is not optimised towards a high speed. Other codes with lower inherent accuracy may easily run a factor 10 faster, and further improvements are possible. Nevertheless, the code is suitable for an interactive work even on a small PC with execution times of a few seconds for the models considered in this paper.

\section{References}

André, P., Bacmann, A., Motte, F., \& Ward-Thompson, D. 1999, in The Physics and Chemistry of the Interstellar Medium, ed. V. Ossenkopf, J. Stutzki, \& G. Winnewisser (GCA-Verlag Herdecke), 241

Auer, L. H. 1987, in Numerical Radiative Transfer, ed. W. Kalkofen (Cambridge Univ. Press), 101

Basu, S., \& Mouschovias, T. C. 1995, ApJ, 452, 386

Basu, S., \& Mouschovias, T. C. 1995, ApJ, 453, 271

Batrla, W., Wilson, T. L., Ruf, K., \& Bastien, P. 1983, A\&A, 128,279

Bodenheimer, P., \& Sweigart, A. 1968, ApJ, 152, 515

Bergin, E. A., \& Langer, W. D. 1997, ApJ, 486, 316

Bonnor, W. B. 1956, MNRAS, 116, 351

Castets, A., \& Langer, W. D. 1995, A\&A, 294, 835

Cesaroni, R., \& Wilson, T. L. 1994, A\&A, 281, 209

Chini, R., Reipurth, B., Ward-Thompson, D., et al. 1997, ApJ, 474, L135

Dickel, H. R., \& Auer, L. H. 1994, ApJ, 437, 222

Fuller, G. A., \& Myers, P. C. 1992, ApJ, 384, 523

Galli, D., \& Shu, F. H. 1993, ApJ, 417, 243

Galli, D., Lizano, S., Li, Z.-Y., Adams, F. C., \& Shu, F. H. 1999, ApJ, 521, 630

Garay, G., \& Lizano, S. 1999, PASP, 111, 1049

Garden, R. P., \& Carlstrom, J. E. 1992, ApJ, 392, 602

Gaume, R. A., Johnston, K. J., \& Wilson, T. L. 1992, ApJ, 388,489

Giannakopoulou, J., Mitchell, G. F., Hasegawa, T. I., Matthews, H. E., \& Maillard, J. 1997, ApJ, 487, 346
Goldsmith, P. F., \& Mao, X.-J. 1983, ApJ, 265, 791

Goodman, A. A., Barranco, J. A., Wilner, D. J., \& Heyer, M. H. 1998, ApJ, 504, 223

Haschick, A. D., \& Ho, P. T. P. 1983, ApJ, 267, 638

Hatchell, J., Thompson, M. A., Millar, T. J., \& Macdonald, G. H. 1998, A\&A, 338, 713

Helmich, F. P., van Dishoeck, E. F., \& Jansen, D. J. 1996, A\&A, 313, 657

Ho, P. T. P., Peng, Y., Torrelles, J. M., et al. 1993, ApJ, 408, 565

Hogerheijde, M. R., van Dishoeck, E. F., Salverda, J. M., \& Blake, G. A. 1999, ApJ, 513, 350

Jaffe, D. T., Davidson, J. A., Dragovan, M., \& Hildebrand, R. H. 1984, ApJ, 284, 637

Jackson, J. M., \& Kraemer, K. E. 1994, ApJ, 429, L37

Keto, E. R. 1990, ApJ, 350, 722

Keto, E. R., \& Ho, P. T. P. 1989, ApJ, 347, 349

Krügel, E., Güsten, R., Schulz, A., \& Thum, C. 1987, A\&A, 185,283

Lada, E. A., Bally, J., \& Stark, A. A. 1991, ApJ, 368, 432

Lada, E. A., Evans, N. J., \& Falgarone, E. 1997, ApJ, 488, 286

Lang, K. R. 1980, Astrophysical Formulae (Springer-Verlag Berlin), 553

Larson, R. B. 1969, MNRAS, 145, 271

Li, Z.-Y., \& Shu, F. H. 1997, ApJ, 475, 237

Lis, D. C., Keen, J., Dowell, C. D., et al. 1998, ApJ, 509, 299

Liseau, R., Lorenzetti, D., Molinari, S., et al. 1995, A\&A, 300, 493

Martin, H. M., Sanders, D. B., \& Hills, R. E. 1984, MNRAS, 208,35

McMullin, J. P., Mundy, L. G., Wilking, B. A., Hezel, T., \& Blake, G. A. 1994, ApJ, 424, 222

Mezger, P. G., Sievers, A. W., Haslam, C. G. T., et al. 1992, A\&A, 256, 631

Miesch, M. S., \& Bally, J. 1994, ApJ, 429, 645

Montalban, J., Bachiller, R., Martin-Pintado, J., Tafalla, M., \& Gomez-Gonzalez, J. 1990, A\&A, 233, 527

Mundy, L. G., Evans, N. J., Snell, R. L., Goldsmith, P. F., \& Bally, J. 1986, ApJ, 306, 670

Myers, P. C. 1999, in The Physics and Chemistry of the Interstellar Medium, ed. V. Ossenkopf, J. Stutzki, \& G. Winnewisser (GCA-Verlag Herdecke), 227

Nakano, M., \& Yoshida, S. 1986, PASJ, 38, 531

Plume, R., Affe, D. T., Evans, N. J., Martin-Pintado, J., \& Gomez-Gonzalez, J. 1997, ApJ, 476, 730

Press, W. H., Teukolsky, S. A., Vetterling, W. T., \& Flannery, B. P. 1992, Numerical Recipies in FORTRAN (Cambridge Univ. Press), 402

Ossenkopf, V. 1997, New Astron., 2, 365

Roberts, D. A., Crutcher, R. M., \& Troland, T. H. 1997, ApJ, 442,208

Rudolph, A., Welch, W. J., Palmer, P., \& Dubrulle, B. 1990, ApJ, 363, 528

Roberts, D. A., Crutcher, R. M., \& Troland, T. H. 1997, ApJ, 479,318

Schneider, N., Simon, R., Kramer, C., Stutzki, J., \& Winnewisser, G. 1999, in The Physics and Chemistry of the Interstellar Medium, ed. V. Ossenkopf, J. Stutzki, \& G. Winnewisser (GCA-Verlag Herdecke), 128

Schulz, A., Güsten, R., Zylka, R., \& Serabyn, E. 1991, A\&A, 246,570

Scoville, N. Z., \& Kwan, J. 1976, ApJ, 206, 718

Shu, F. H. 1977, ApJ, 214, 488 
Sievers, A. W., Mezger, P. G., Kreysa, E., et al. 1991, A\&A, Turner, B. E., Chan, K.-W., Green, S., \& Lubowich, D. A. 251,231

Sobolev, V. V. 1957, Soviet. Astron., 1, 678

Stutzki, J., \& Winnewisser, G. 1985, A\&A, 144, 13

Stutzki, J., Bensch, F., Heithausen, A., Ossenkopf, V., \& Zielinsky, M. 1998, A\&A, 336, 697

Tieftrunk, A. R., Wilson, T. L., Steppe, H., et al. 1995, A\&A, 303, 901

Tieftrunk, A. R., Gaume, R. A., Claussen, M. J., Wilson, T. L., \& Johnstone, K. J. 1997, A\&A, 318, 931 1992, ApJ, 399, 114

Welch, W. J., Dreher, J. W., Kackson, J. M., Terebey, S., \& Vogel, S. N. 1987, Science, 238, 1550

White, R. E. 1977, ApJ, 211, 744

Wiesemeyer, H., Güsten, R., Wink, J. E., \& Yorke, H. W. 1997, A\&A, 320, 287

Wilson, T. L., Johnston, K. J., \& Mauersberger, R. 1991, A\&A, 251, 220

Wood, D. O. S., \& Churchwell, E. 1989, ApJS, 69, 831

Tieftrunk, A. R., Gaume, R. A., \& Wilson, T. L. 1998, A\&A, 340,232

Young, L. M., Keto, E., \& Ho, P. T. P. 1998, ApJ, 507, 270

Zang, Q., \& Ho, P. T. P. 1997, ApJ, 488, 241 